\title{
THE GLOBAL KINEMATICS OF THE GLOBULAR CLUSTER M92
}

\author{
G. A. DrukIER \\ Department of Astronomy, Yale University, New Haven, CT, USA; drukier@astro.yale.edu \\ H. N. Cohn AND P. M. LugGer \\ Department of Astronomy, Indiana University, Bloomington, IN, USA; cohn@astro.indiana.edu, lugger@astro.indiana.edu \\ S. D. Slavin \\ Department of Chemistry and Physics, Purdue University Calumet, Hammond, IN, USA; slavin@calumet.purdue.edu \\ R. C. BERRington \\ Department of Physics and Astronomy, University of Wyoming, Laramie, WY, USA; rberring@uwyo.edu \\ AND \\ B. W. MuRPHY \\ Department of Physics and Astronomy, Butler University, Indianapolis, IN, USA; bmurphy@butler.edu \\ Received 2005 July 26; accepted 2006 November 5
}

\begin{abstract}
We report the determination of high-accuracy radial velocities for 306 members of the globular cluster M92 using the Hydra multiobject spectrograph on the WIYN telescope. We have concentrated on stars outside of the central region of the cluster, located up to $14.4^{\prime}$ from the cluster center. Candidate members were selected for spectroscopy based on a photometric metallicity index determined from three-band Washington photometry, also obtained with the WIYN telescope. The median error in the velocities is $0.35 \mathrm{~km} \mathrm{~s}^{-1}$. We find the heliocentric radial velocity of the cluster to be $-121.2 \pm 0.3 \mathrm{~km} \mathrm{~s}^{-1}$. We have used an improved Bayesian analysis to determine the velocity dispersion profile of M92. The most probable profile is a cored power law with a scale radius of $2^{\prime}$, a velocity dispersion at $1^{\prime}$ of $6.3 \mathrm{~km} \mathrm{~s}^{-1}$, and an outer power law with a slope of -0.6 . We have also reanalyzed the M15 radial velocities of Drukier et al. and find that a pure power law with a $1^{\prime}$ velocity dispersion of $8 \mathrm{~km} \mathrm{~s}^{-1}$ and a slope of -0.5 and the combination of a power law with a slope of -0.4 and a scale of $7.5 \mathrm{~km} \mathrm{~s}^{-1}$ inside $9^{\prime}$ and a dispersion of $4 \mathrm{~km} \mathrm{~s}^{-1}$ outside are equally likely. In both clusters there is evidence that the samples include escaping stars. We present results from a GRAPE-based $N$-body simulation of an isolated cluster that demonstrates this effect. We suggest additional tests to determine the relative importance of tidal heating and stellar ejection for establishing the velocity field in globular cluster halos.
\end{abstract}

Key words: globular clusters: individual (M92, M15) — methods: statistical — stellar dynamics

Online material: machine-readable tables

\section{INTRODUCTION}

In globular clusters, the interplay between two-body relaxation and external tidal stresses is most obvious in their outer parts. There has been considerable recent interest in the evolution and eventual dissolution of clusters in the Galactic tidal field (e.g., Gnedin et al. 1999; Combes et al. 1999; Takahashi \& Portegies Zwart 2000; Dehnen et al. 2004). To investigate these issues, we have been carrying out a program to determine the global velocity fields of globular clusters using the Hydra fiber-fed, multiobject spectrograph on the WIYN telescope. ${ }^{1}$ With its $1^{\circ}$ diameter field and echelle grating, Hydra is well suited for determining highaccuracy radial velocities of stars out to the tidal radii of clusters. Our approach is an important complement to Fabry-Pérot imaging (e.g., Gebhardt et al. 1994, 2000), which can only be used efficiently in the central $1^{\prime}-2^{\prime}$.

In a previous paper (Drukier et al. 1998, hereafter Paper I) we reported a new global velocity dispersion profile for the prototypical collapsed-core cluster M15. We found a clear indication of a flattening and possible rise of the profile in the outer part of

\footnotetext{
${ }^{1}$ The WIYN Observatory is a joint facility of the University of Wisconsin, Indiana University, Yale University, and the National Optical Astronomy Observatory.
}

the cluster. In contrast, our anisotropic Fokker-Planck simulations of isolated clusters show a smoothly declining velocity dispersion profile (Drukier et al. 1999). We interpreted our observations of M15 as evidence for heating of the cluster halo by the Galactic tidal field. Johnston et al. (1999) subsequently showed that a Galactic satellite system undergoing tidal heating will show a break in the slope of the velocity dispersion profile at the radius at which unbound stars begin to dominate. However, this leaves open the question of whether two-body relaxation in an isolated cluster could produce a similar effect through strong scattering of stars from the central region into unbound orbits. Neither our Fokker-Planck simulations nor the self-consistent field simulations of Johnston et al. (1999) include strong scattering. We address this question in the present study through direct $N$-body integrations, which include all scattering mechanisms.

We chose M92 (NGC 6341) for the second cluster in our survey, as a "normal" comparison to the collapsed-core cluster M15. The properties of these two clusters are compared in Table 1. Unlike M15, M92 has a well-resolved core. While its central luminosity density is in the upper third of the distribution for all Galactic globular clusters (Harris 1996), it is nevertheless at least an order of magnitude lower than that of M15. Both clusters are brighter than the median of the absolute magnitude distribution; M92 is in the upper $30 \%$, while M15 is in the upper decile. Both 
TABLE 1

Comparison of M92 And M15

\begin{tabular}{|c|c|c|}
\hline Property $^{\text {a }}$ & M92 & M15 \\
\hline$M_{V} \ldots \ldots \ldots \ldots \ldots \ldots \ldots$ & -8.20 & -9.17 \\
\hline $\log \rho_{0}\left(L_{\odot} \mathrm{pc}^{-3}\right)$ & 4.29 & $>5.38$ \\
\hline 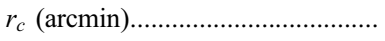 & 0.23 & $<0.025^{\mathrm{b}}$ \\
\hline 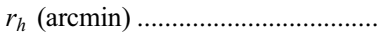 & 1.09 & 1.06 \\
\hline$r_{t}(\operatorname{arcmin}) .$. & 15.2 & 21.5 \\
\hline$c \ldots \ldots \ldots \ldots \ldots \ldots$ & 1.81 & $>2.5$ \\
\hline$[\mathrm{Fe} / \mathrm{H}] \ldots \ldots \ldots \ldots \ldots$ & -2.29 & -2.25 \\
\hline 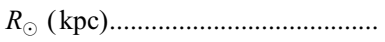 & 8.2 & 10.3 \\
\hline 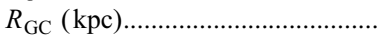 & 9.6 & 10.4 \\
\hline ……................. & 4.7 & -4.7 \\
\hline
\end{tabular}

Notes. $-M_{V}$, total absolute magnitude; $\rho_{0}$, central luminosity density; $r_{c}$, core radius; $r_{h}$, half-mass radius; $r_{t}$, tidal radius; $c$, concentration parameter; $[\mathrm{Fe} / \mathrm{H}]$, metallicity; $R_{\odot}$, solar distance; $R_{\mathrm{GC}}$, galactocentric distance; $Z$, distance from Galactic plane.

a These data are from the online compilation of Harris (1996), http://www.physics.mcmaster.ca/ harris/mwgc.dat.

b Sosin \& King (1997).

are among the very lowest metallicity clusters. Both are located approximately $10 \mathrm{kpc}$ from the Galactic center and nearly $5 \mathrm{kpc}$ from the Galactic plane. Both are part of the oldest group of clusters (Chaboyer et al. 1996).

The radial velocity of M92 $\left(-121.2 \pm 0.3 \mathrm{~km} \mathrm{~s}^{-1}\right)$ and its very low metallicity $([\mathrm{Fe} / \mathrm{H}]=-2.29)$ clearly distinguish its stars from the bulk of the field stars in the same region of the sky. Nevertheless, selecting likely cluster members for spectroscopy becomes challenging in the outer halo, where the vast majority of stars are nonmembers. As we pointed out in Paper I, selecting stars based on their position in a color-magnitude diagram (CMD) alone is not an efficient means of finding potential members. As we discuss in $\S 2.1$, we turned to a metallicity-sensitive colorcolor diagram in the Washington system (Canterna 1976) to aid our candidate selection.

There have been a number of previous velocity studies of M92. The most extensive is that of Lupton et al. (1985), who observed 49 stars in M92 using the $5 \mathrm{~m}$ Hale telescope, but, unfortunately, the individual velocities have not been published. Lupton et al. (1985) found little sign of anisotropy and only a weak ( $2 \sigma$ at best) rotational signal. The dispersion profile itself was fairly flat, but the statistics in the outer part of the cluster were particularly poor. Beers et al. (1990) observed only seven stars, and the uncertainty in the velocities was $7 \mathrm{~km} \mathrm{~s}^{-1}$. While the present study was under way, Soderberg et al. (1999) reported highprecision measurements for 35 stars in M92, based on Hydra spectroscopy with the Kitt Peak National Observatory (KPNO) $4 \mathrm{~m}$ telescope and the WIYN telescope in the region about the $\mathrm{Na} \mathrm{D}$ line. Thus, while some previous radial velocity information is available for M92, it is not sufficient for a determination of the global velocity dispersion profile, particularly in the cluster halo. Good-quality proper motions for M92 stars are available for of order 700 stars located out to $19^{\prime}$ from the cluster center (Rees 1992; Tucholke et al. 1996).

As in Paper I, we carried out our observations on the $3.5 \mathrm{~m}$ WIYN telescope. We used the Hydra fiber-fed, multiobject spectrograph to obtain high-resolution spectra. This resulted in a homogeneous set of over 300 M92 stars with new, high-accuracy velocity measurements.

In the absence of a full dynamical study, data sets such as these are customarily analyzed by binning the stars in radius and computing the dispersion in their velocities. In this way an approx- imation of the dispersion profile is constructed. In this paper we use the methods of Bayesian statistics to move beyond this in three ways. First, besides the traditional bins, we also examine several continuous dispersion profiles, as described in $\S 3.2$. Second, our methods allow us to assess which of the several candidate profiles is best supported by the data. Third, by looking at the posterior probability distributions for the parameters we can determine the extent to which they deviate from Gaussian distributions.

We discuss our observations in $\S 2$. It particular, we review our new photometric selection technique using the Washington system. In $\S 4$ we use the Bayesian techniques described in $\S 3$ to analyze the velocity distributions of both the new M92 data and the M15 data from Paper I. We then do a similar analysis of an $8 \mathrm{~K} N$-body model to investigate the effects of unbound stars on the dispersion analysis. We conclude with a summary of the main results of this paper and a suggestion of some directions for further progress in investigating the questions raised by these data sets.

\section{OBSERVATIONS}

\subsection{Photometry and Candidate Selection}

In order to observe with Hydra, it is necessary to prepare a list of target stars with accurate positions. For this project, the targets are those stars found on the giant branch that are likely to be cluster members. Our candidate list comes from three sources. For our 1996 May run and the first night of the 1996 June run, our candidates all came from the proper-motion list of Rees (1992). We selected stars appearing on the $V$ versus $B-V$ CMD but did not select on the basis of the assigned membership probabilities. For the bulk of the 1996 June run, our stars were selected from a list of stars found on KPNO Burrell Schmidt CCD images kindly supplied by H. Morrison. The images had been taken in the Washington system, which we discuss further below. Owing to problems with obtaining sufficiently accurate photometry from these images, we selected stars only on the basis of their CMD position, rather than using the color-color plot, which provides metallicity information. We observed 444 stars in 1996 and obtained useful velocities for 427 of these. Only 192 stars $(45 \%)$ proved to be members.

As we discussed in Paper I, selecting stars on the basis of a CMD alone is not the most efficient method of finding likely members. Depending on the distance and Galactic latitude of the cluster, contamination by Galactic dwarfs with the colors of the cluster giant branch can be significant. This is a particular problem in the outer part of a globular cluster, where the members are sparse on the sky and are outnumbered by nonmembers even at high Galactic latitudes. A better method is to take advantage of the metallicity difference between a globular cluster and the Galactic field.

The Washington system (Canterna 1976) is designed to determine stellar metallicities along the giant branch. It consists of four broadband filters, $C, M, T_{1}$, and $T_{2}$. The last is equivalent to Kron-Cousins $I$. The $T_{1}-T_{2}$ color was originally intended to be the temperature index, with $C-M$ giving the metallicity. The $C$ band was selected to occupy the region around $400 \mathrm{~nm}$ occupied by the $\mathrm{Ca} \mathrm{H}$ and $\mathrm{K}$ doublet, the $\mathrm{G}$ band, and plentiful $\mathrm{CN}$ bands in these cool stars. The $M$ band is in a spectral region centered near $510 \mathrm{~nm}$ that is less affected by metallicity. Geisler et al. (1991) have shown that the optimal diagram for metallicity determination is the $C-M$ versus $M-T_{2}$ color-color diagram. This gives us the maximum metallicity sensitivity and only requires observation in three broadband colors.

In 1997 May we observed M92 using the imager on the WIYN telescope. We observed a 40 field mosaic covering a $15^{\prime}$ radius 


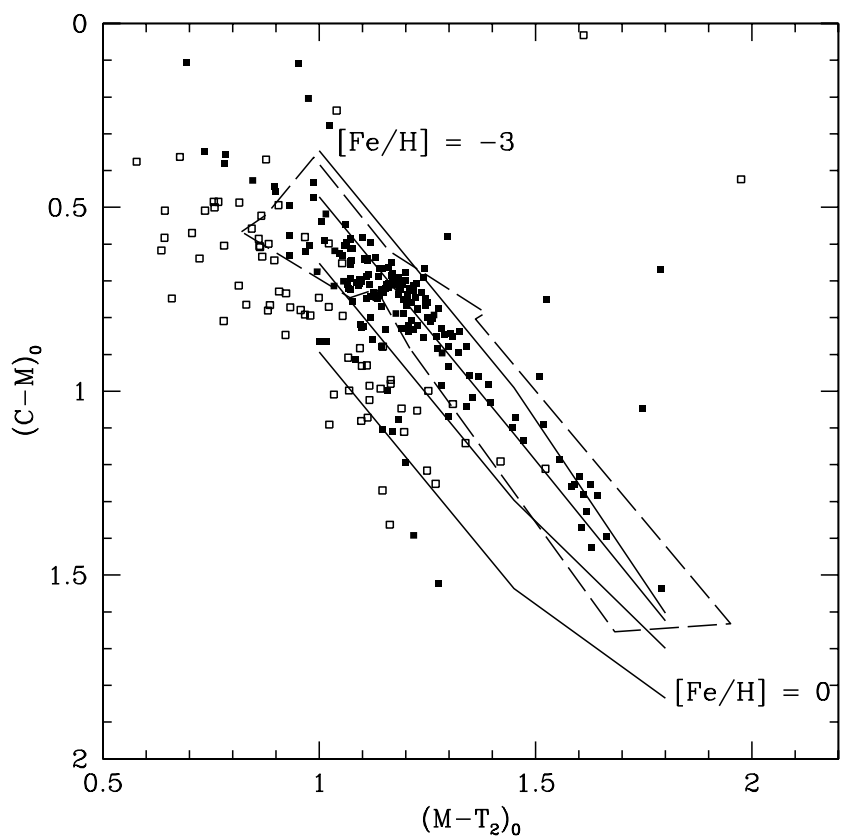

Fig. 1.-Washington system $C-M$ vs. $M-T_{2}$ color-color diagram for the stars observed in 1996. Only stars with the higher precision WIYN photometry are shown. The filled symbols represent the stars that were subsequently determined to be members of M92, and the open symbols represent nonmembers. The solid lines indicate loci of common metallicity from Geisler et al. (1991). Based on this diagram, we selected the region within the dashed lines for our 1997 observations.

centered on the cluster, with a few fields extending out to $20^{\prime}$. We observed each field with the $C, M$, and $T_{2}$ filters. We also observed using the DDO 51 filter as suggested by Geisler (1984). This should have allowed us to separate dwarfs from giants on the basis of the luminosity effects in the $\mathrm{Mg} b$ triplet. The DDO 51 photometry lacked sufficient precision to permit this, possibly due to error propagation in transferring the zero point across the large mosaic. The DDO 51 frames were used for the astrometry described below.

Each frame was debiased and flat-fielded in the usual manner, and photometry was done on each using DAOPHOT II and ALLSTAR (Stetson 1987; Stetson \& Harris 1988). Since the imaging was not done under photometric conditions, all of the photometry was put on a common zero point by matching stars in overlapping regions between frames. In a subsequent photometric run in 1997 October, we observed one field in M92, as well as a region in standard field SA 114 containing three standard stars from Geisler (1996). This allowed us to put our $C, M$, and $T_{2}$ observations onto the standard system. Having only the one standard image, we used the first-order extinction coefficients from Harris \& Canterna (1979). Our selection of likely cluster members, however, was based on the uncalibrated color-color diagram.

Our original region, selected to include the giant branch, was $M<18, C-M>0.2$, and $M-T_{2}>0.5$. The stars observed in 1996 with photometry from our WIYN mosaic are shown in Figure 1 in the calibrated $C-M$ versus $M-T_{2}$ color-color diagram. We have adopted $E(B-V)=0.02$ (Harris 1996) for M92 and taken the reddening ratios from Harris \& Canterna (1979). These stars were selected on the basis of their position in the CMD. The open symbols represent the nonmembers, and the filled symbols represent the spectroscopically determined members. We have also included the loci of the $[\mathrm{Fe} / \mathrm{H}]=0$ through $[\mathrm{Fe} / \mathrm{H}]=$ -3 stars, in 1 dex steps in $[\mathrm{Fe} / \mathrm{H}]$, from Figure 7 of Geisler et al. (1991). The separation by metallicity is quite clear. We based our

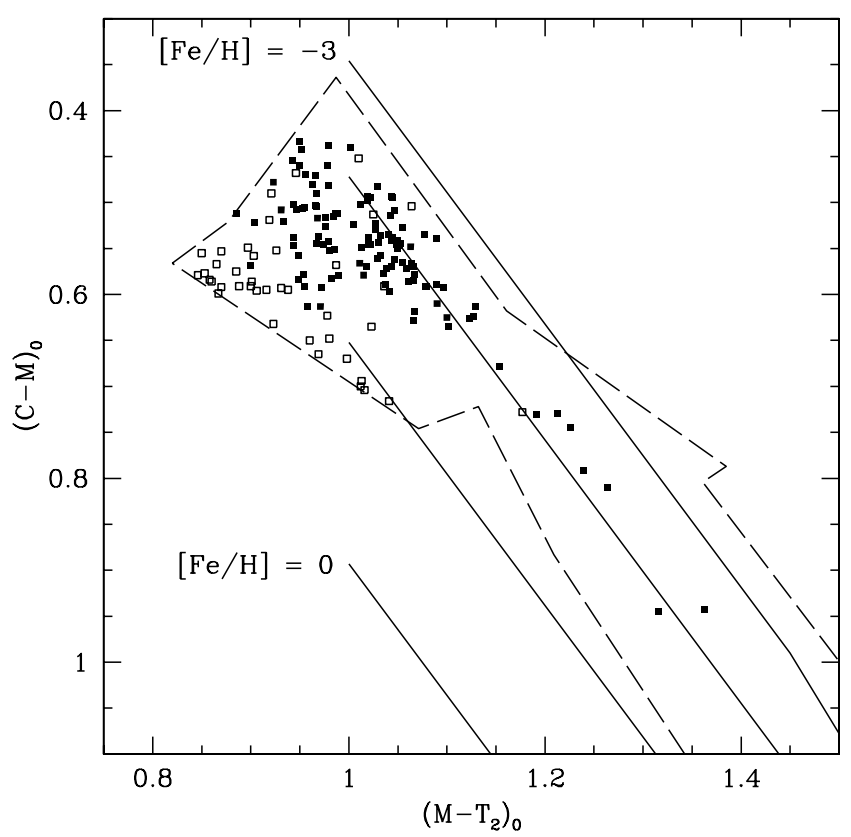

FIG. 2.- Same as Fig. 1, but for the stars observed in 1997. The stars in this sample were selected only on the basis of their positions in this diagram.

final selection of stars for the 1997 observing season on their location in this diagram. The selected region is indicated by the dashed box.

Figure 2 shows the stars observed in the 1997 observing season. The lines and symbols are the same as in Figure 1. These stars were primarily selected to lie outside of $3^{\prime}$. We observed 162 new stars based on their Washington colors, and 152 yielded useful velocities. Of these stars, $111(73 \%)$ proved to be members. Outside $3^{\prime}$, we measured velocities for 140 stars. Of these, $99(71 \%)$ were members. By contrast, for stars observed in 1996 and defined the same way $\left(r>3^{\prime}\right.$ and with subsequent WIYN photometry), only 59 of the 142 stars $(42 \%)$ proved to be members, although they were, admittedly, selected on the basis of an inferior CMD. Nonetheless, the 1997 sample extended to a larger distance from the cluster center and would have had a lower success rate had the candidates been selected in the CMD alone. Figure 3 shows the $M$ versus $M-T_{2}$ CMD with members and nonmembers distinguished. Most of the stars in the 1997 sample (Fig. 2) have $M>15.7$ and $M-T_{2}<1.2$ in this CMD, while most of the stars with $M<15.5$ are from the 1996 sample (Fig. 1). A comparison between the two samples makes it clear that member selection is easier in the Washington color-color diagram.

\subsection{Astrometry}

We calculated the astrometric positions of our stars using version 1.2 of the Hubble Space Telescope (HST) Guide Star Catalog (GSC) as our astrometric system (see Lasker et al. 1990). About 9000 stars in a $45^{\prime} \times 45^{\prime}$ region around M92 from the Quick $V$ Palomar Schmidt survey were used as secondary astrometric standards. Plate solutions were calculated for each of the DDO 51 WIYN frames in the mosaic; the narrowband filter avoids any problems with differential refraction. The astrometric solution was good to better than the $0.3^{\prime \prime}$ required for Hydra.

\subsection{Velocity Observations}

We obtained all observations with the same spectrograph configuration we used for M15 (Paper I). We used the echelle grating with an order centered at $515 \mathrm{~nm}$, in the neighborhood of the 


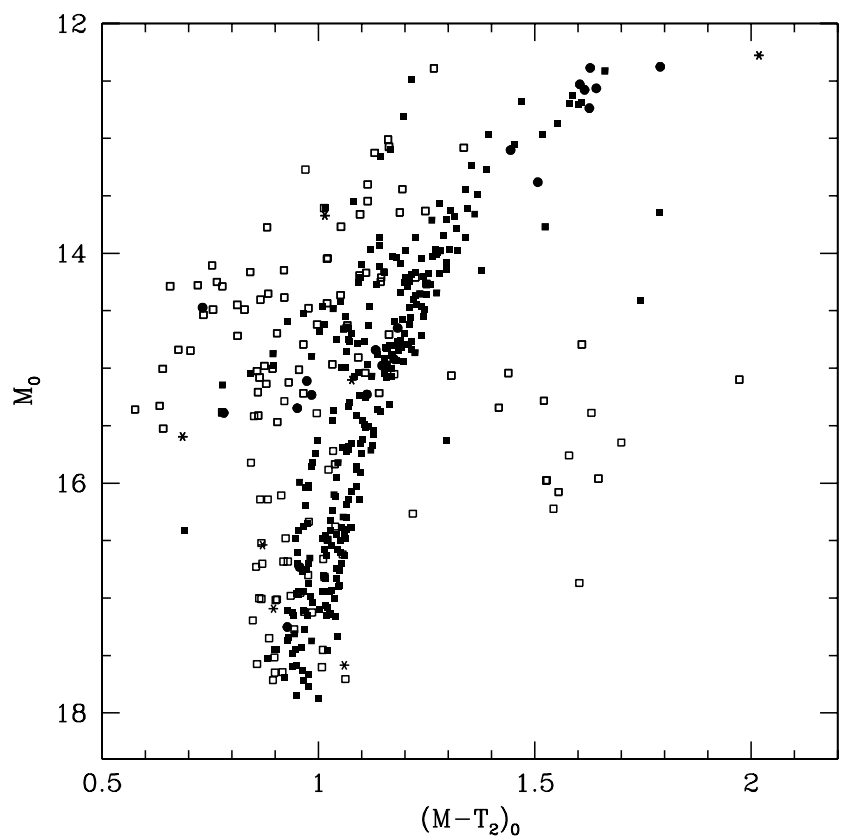

Fig. 3.-Washington system CMD for all stars with measured velocities. The filled symbols represent confirmed members, with circles for the velocity variables and squares for the nonvariables. The open squares represent nonmembers, and the asterisks represent doubtful members. Most of the stars brighter than $M=15.5$ were observed in 1996; those fainter are from the color-selected 1997 sample.

$\mathrm{Mg} b$ lines. Approximately $20 \mathrm{~nm}$ of the order was imaged on the 2048 pixel long CCD for a dispersion of about $0.01 \mathrm{~nm} \mathrm{pixel}^{-1}$. The comparison source was a Th-Ar lamp. The Hydra multifiber positioner and WIYN bench spectrograph were used. The positions for the sky fibers were determined from the Quick $V$ frame.

We observed M92 over the course of four observing runs in 1996 May, 1996 June, 1997 June, and 1998 June. Table 2 contains a log of the observations, including the number of stars observed in each configuration. The 1996 May runs observed fewer stars than the later runs, since the proper-motion list of Rees (1992) was confined to the inner $13.3^{\prime}$ of the cluster. The Schmidt images and the WIYN data covered a much larger area, allowing us to observe more stars per configuration. As the 1997 June run progressed, we did a rough reduction of each night's data and removed stars showing grossly different velocities from that of the cluster. This allowed us to concentrate on the stars more likely to be members. Most of the removed stars were in the outer part of the cluster; so, since it is harder to place fibers in the more crowded inner region, the number of stars observed per configuration went down as each run progressed. The proportion of members increased, however. In 1998 June we observed one configuration in order to refine our velocities of a number of stars, but more particularly to obtain additional spectra of a few stars with ambiguous membership.

In all we obtained 1204 useful spectra of 596 stars. Of these, 306 turned out to be definite members, as discussed below. We include in this the total velocities for six faint stars for which we could measure only a single velocity by combining all of their spectra. Five of these turned out to be members. For two of these members an additional, confirming velocity was obtained in 1998 June.

\subsection{Velocity Measurement}

We repeatedly observed three stars, IV-10, III-13, and 220. Star IV-10 was our primary standard and was stable, as was 220, but III-13 showed the classic jitter of bright giants. We used IV-10
TABLE 2

OBSERVING LOG FOR M92

\begin{tabular}{|c|c|c|c|c|}
\hline Configuration & UT Date & HJD & $\begin{array}{c}\text { Exposure Time } \\
\text { (s) }\end{array}$ & No. of Stars \\
\hline $\mathrm{m} 1 \ldots$ & 1996 May 14 & $10,217.799$ & 2400 & 38 \\
\hline $\mathrm{m} 2 \ldots \ldots \ldots \ldots \ldots$ & 1996 May 16 & $10,219.757$ & 1800 & 26 \\
\hline 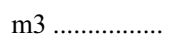 & 1996 May 17 & $10,220.764$ & 1800 & 27 \\
\hline $\mathrm{m} 4$ & 1996 May 17 & $10,220.852$ & 3000 & 24 \\
\hline m5 ……............... & 1996 May 18 & $10,221.769$ & 3000 & 32 \\
\hline m6 .................... & 1996 May 19 & $10,222.743$ & $4200^{\mathrm{a}}$ & 29 \\
\hline $\mathrm{m} 7$................... & 1996 May 19 & $10,222.845$ & 3600 & 31 \\
\hline M6 ..... & 1996 Jun 19 & $10,253.685$ & 1800 & 29 \\
\hline M7 .................. & 1996 Jun 19 & $10,253.740$ & 1800 & 32 \\
\hline M8 ................... & 1996 Jun 19 & $10,253.785$ & 1800 & 31 \\
\hline $\mathrm{J} 1$........................ & 1996 Jun 21 & $10,255.687$ & 1800 & 88 \\
\hline $\mathrm{J} 2 \ldots \ldots \ldots \ldots \ldots \ldots$ & 1996 Jun 21 & $10,255.747$ & 1800 & 87 \\
\hline $\mathrm{J} 3 \mathrm{a} \ldots \ldots \ldots \ldots \ldots \ldots$ & 1996 Jun 21 & $10,255.818$ & $991^{\mathrm{a}}$ & 85 \\
\hline $\mathrm{J} 3 \mathrm{~b} \ldots \ldots \ldots \ldots \ldots \ldots$ & 1996 Jun 23 & $10,256.724$ & 1800 & 85 \\
\hline $\mathrm{J} 4$....................... & 1996 Jun 23 & $10,256.793$ & 1800 & 77 \\
\hline J5 „................... & 1996 Jun 23 & $10,257.803$ & 1800 & 66 \\
\hline A & 1997 Jun 22 & $10,621.724$ & 7200 & 71 \\
\hline В ……................. & 1997 Jun 22 & $10,621.875$ & 7200 & 69 \\
\hline $\mathrm{C} \ldots \ldots \ldots \ldots \ldots \ldots$ & 1997 Jun 23 & $10,622.727$ & 7200 & 60 \\
\hline 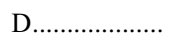 & 1997 Jun 23 & $10,622.888$ & 6760 & 57 \\
\hline E ….................... & 1997 Jun 24 & $10,623.708$ & 7200 & 57 \\
\hline F …..................... & 1997 Jun 24 & $10,623.833$ & 7200 & 53 \\
\hline G. & 1997 Jun 25 & $10,624.723$ & 7200 & 54 \\
\hline 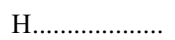 & 1997 Jun 25 & $10,624.882$ & 7200 & 58 \\
\hline K....................... & 1997 Jun 26 & $10,625.725$ & 7200 & 55 \\
\hline ............... & 1997 Jun 26 & $10,621.853$ & 7200 & 54 \\
\hline M & 1998 Jun 16 & $10,980.690$ & 7200 & 69 \\
\hline
\end{tabular}

a Observation affected by clouds.

as our velocity template for the velocity determination by crosscorrelation.

The data were reduced using the dohydra reduction package in IRAF. ${ }^{2}$ Each observation was accompanied by one or more 5 minute exposures of an incandescent lamp (a "flat") taken with the fibers in the same configuration as the observations. Generally, configurations observed at the end of the night had multipleflat exposures, but, because of the overhead involved with flats and especially with reconfiguring the fibers, we usually only took a single-flat exposure. There did not appear to be any disadvantage to using single-flat exposures, since cosmic rays were not a great problem. The program exposures and bracketing Th-Ar lamp exposures were extracted and then divided by the extracted lamps. In the two 1996 runs and the 1998 observation no sky subtraction was required, since they were observed in dark time. Our 1997 June run took place in brighter conditions, and some sky subtraction was required. The sky contribution was small and only affected the observations at the beginning of each night.

Cosmic-ray removal was done through the simple expedient of using the IRAF continuum task to replace all pixels more than 4 standard deviations above the fit with the continuum fit. Unlike in Paper I, we did the cosmic-ray removal before the dispersion correction and resampling. This prevented the negative spikes we saw in the M15 data.

As in our M15 observations, the wavelength calibration was done using 34-36 comparison lines. The fifth-order dispersion solution generally had rms residuals of less than $10^{-4} \mathrm{~nm}$ or

\footnotetext{
${ }^{2}$ IRAF is distributed by the National Optical Astronomy Observatory, which is operated by the Association of Universities for Research in Astronomy, Inc., under cooperative agreement with the National Science Foundation.
} 


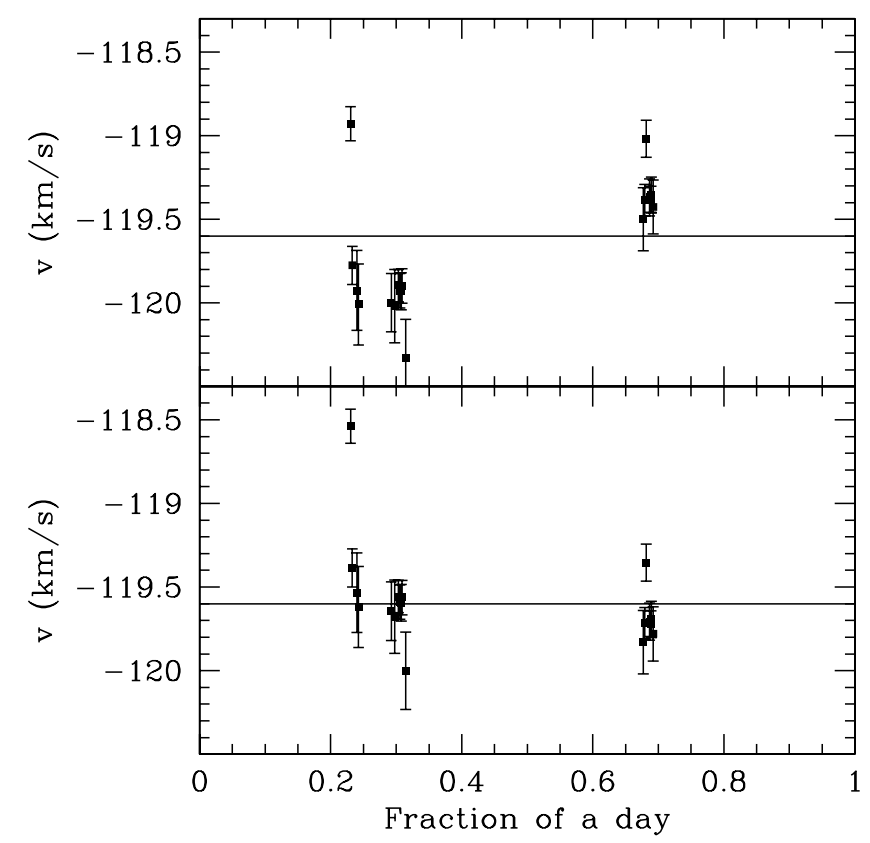

FIG. 4.-Top: Relative velocity of twilight-sky exposures corrected for all motions except rotation of the Earth plotted against the fraction of a day from mean noon at Kitt Peak. Bottom: After correction for terrestrial rotation.

$0.05 \mathrm{~km} \mathrm{~s}^{-1}$. During dispersion correction the spectra were resampled into 2048 logarithmically spaced bins covering $20.7 \mathrm{~nm}$ in total.

In 1996 May, 1997 June, and 1998 June we took multiple exposures in each configuration. The resulting spectra for each star from each configuration were summed together to produce the final spectra for cross-correlation. The velocities were calculated using the fxcor task in IRAF. As in Paper I, we excluded all spectra with cross-correlation peaks smaller than 0.2 . These had low signal-to-noise ratios and gave highly discordant velocities in most cases. The template consisted of the sum of all exposures of star IV-10 in 1996 and 1997 suitably shifted to a common heliocentric velocity. This spectrum is the result of $28 \mathrm{hr}$ of total integration time.

Our error analysis proceeded as in Paper I, following the method of Pryor et al. (1988). We used the repeated observations of stars to establish the value for the constant in the Tonry \& Davis (1979) formula for the velocity uncertainty. In this instance we obtained $C=12.8 \pm 0.5 \mathrm{~km} \mathrm{~s}^{-1}$. This is quite consistent with the value of $13.1 \pm 0.5 \mathrm{~km} \mathrm{~s}^{-1}$ we obtained for M15 in Paper I. As the experimental setup is the same and M15 and M92 have similar metallicities, this is not surprising.

The velocity zero point was established using 18 spectra of the twilight sky taken during various observing runs. During our 1997 June run, sky observations were taken at both evening and morning twilight. We extracted the spectra in the usual manner and cross-correlated them against the template spectrum. The individual velocities were examined for fiber-to-fiber variations. Nothing significant was seen. Using the value of $C$ above, the individual velocities have errors of $1 \mathrm{~km} \mathrm{~s}^{-1}$. This is primarily an effect of the mismatch between the low-metallicity template and the solar spectrum of the twilight sky. The mean velocity in each image had a standard deviation of only $0.1-0.2 \mathrm{~km} \mathrm{~s}^{-1}$. These means were used in estimating the zero point.

We noticed a systematic difference between the 1997 June evening and morning exposures of about $0.54 \pm 0.02 \mathrm{~km} \mathrm{~s}^{-1}$, excluding one anomalous value. We attributed this to the rotational

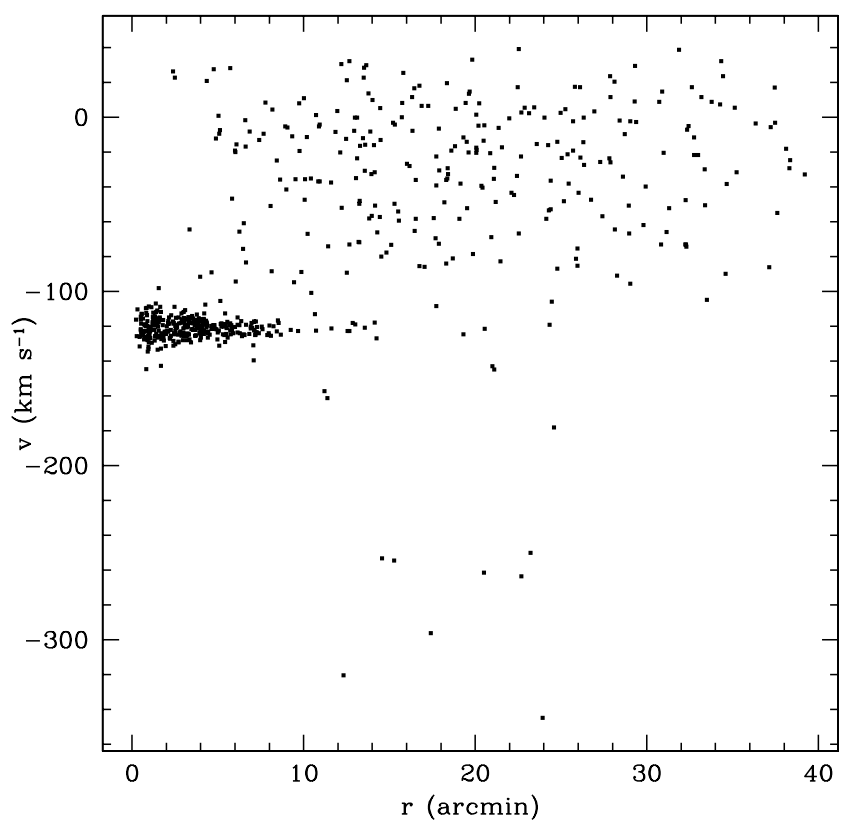

FIG. 5.-Radial velocity vs. distance from the center of the cluster for all observed stars. The cluster near $-120 \mathrm{~km} \mathrm{~s}^{-1}$ is mostly cluster stars. A few presumable halo stars are visible at more negative velocities.

velocity of the Earth, which, at the latitude of Kitt Peak and for the times of the observations, amounts to $0.64 \mathrm{~km} \mathrm{~s}^{-1}$. Subtracting this contribution leaves a difference smaller than the errors in the means for the two twilights. We show the results in Figure 4. The top panel shows the velocities corrected for all motions except the rotation of the Earth against the fraction of a day from mean noon at Kitt Peak. Therefore, we corrected all the sky velocities to the heliocentric frame. There were still some residual differences between evening and morning observations, as seen in the bottom panel of Figure 4. These were $0.07 \mathrm{~km} \mathrm{~s}^{-1}$ from their mean in 1997 June, equivalent to about 0.02 pixels at our dispersion. The highly consistent behavior leads us to suspect some remaining systematic effect we are unable to identify. We are similarly unable to account for the large deviations from the mean of a couple of observations. Our zero point is taken as the unweighted mean of all the corrected sky observations and is $-119.6 \pm 0.1 \mathrm{~km} \mathrm{~s}^{-1}$.

Figure 5 shows the velocities for all the stars observed. The group near $-120 \mathrm{~km} \mathrm{~s}^{-1}$ is principally made up of cluster members; the Galactic disk stars cluster near zero. As in M15 we see a few stars with velocities significantly more negative than the cluster. These are presumably field halo stars.

We established membership by requiring velocity coherence and line strengths appropriate for low-metallicity giants. In Paper I the metallicity criterion was simply an estimate of the equivalent width of the Mg line at $518.3 \mathrm{~nm}$. For our M92 observations, however, there were several stars with weak spectra for which this technique gave ambiguous results. Instead, we have used a variation of the method of Ratnatunga \& Freeman (1989). They showed that the height of the autocorrelation function for a spectrum is related to the star's metallicity. Since we simply desire an uncalibrated measure of the metallicity, we have simplified their procedure somewhat. We have filtered the spectra in the Fourier regime to remove the highest frequencies before autocorrelation and normalized the autocorrelation height by the log of the mean number of counts in the spectrum. We plot this $h_{1}$ index against the radial velocity of the stars in Figure 6. 


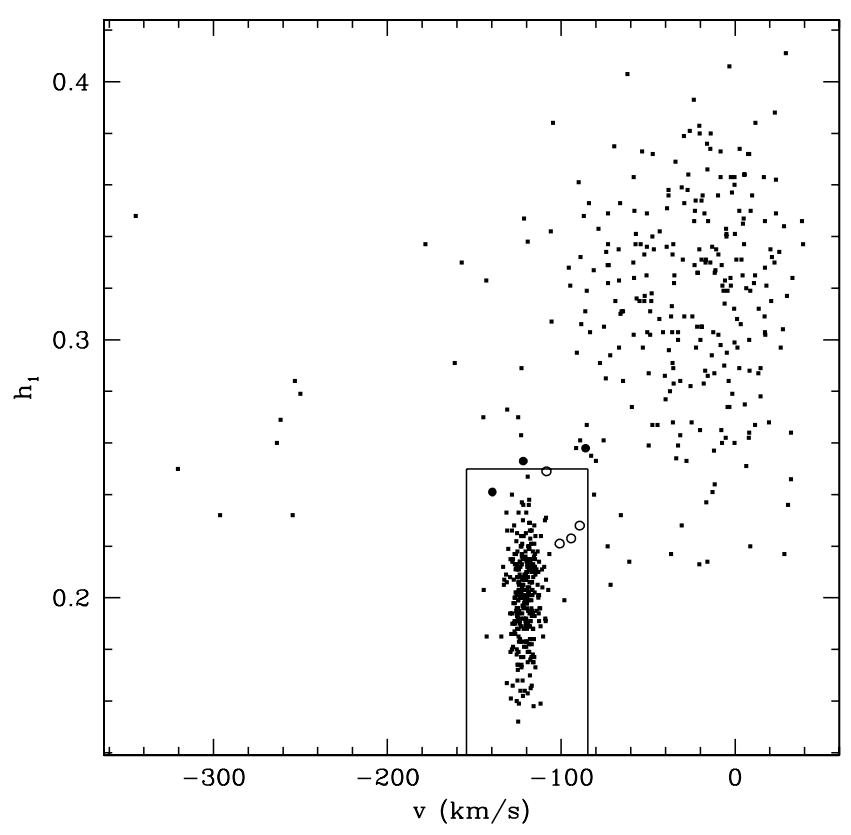

FIG. 6.-Normalized autocorrelation height, $h_{1}$, plotted against velocity. The box contains the locus of the members. The open circles represent the four stars rejected for the reasons discussed in the text. The filled circles represent other doubtful members.

We make the following cuts in Figure 6 to establish our members. First, we reject all stars with velocities differing from the template by more than $35 \mathrm{~km} \mathrm{~s}^{-1}$. Second, we reject all stars with $h_{1}>0.25$. This leaves us with 310 stars within the box in the figure. Of these, four stars (Fig. 6, open circles) have been rejected from consideration for the dispersion analysis. All show highly discrepant velocities compared with other stars at the same distance, and all but one have zero probability of membership based on Tucholke et al. (1996) proper motions. Other stars with zero probability have been included in the sample if they otherwise satisfy the criteria above.

The remaining 306 stars are listed in Table 3. Our J2000.0 coordinates are given in columns (1) and (2), followed by a stellar identification. Where possible, we have used identifications that are generally derived from Table 3 of Tucholke et al. (1996), although we have made some additions and corrections where we have deemed them appropriate. These identifiers primarily come from Sandage \& Walker (1966; hyphenated Roman numeral prefixes or the letter " $x$ "), Cudworth (1976; C), and Buonanno et al. $(1983 ; \mathrm{Bu}){ }^{3}$ with additional stars from Barnard $(1931 ; \mathrm{B})$, Nassau (1938; N), Zinn et al. (1972; ZNG), Sawyer Hogg (1973; V), and Rees (1992; R). Where available, other names from these sources are given in column (11). Where no previous identification exists, we have given our own number. Column (4) contains the distance from the cluster center, which we take as $\alpha(\mathrm{J} 2000.0)=$ $17^{\mathrm{h}} 17^{\mathrm{m}} 07.02^{\mathrm{s}}, \delta(\mathrm{J} 2000.0)=43^{\circ} 08^{\prime} 11.4^{\prime \prime}$. Columns $(5)-(7)$ give our Washington photometry. Column (8) gives the number of velocities for the star. Where there was only a single observation, the identification of the configuration in Table 2 is given. The two stars with configuration " $Z$ " have single velocities based on sums of all the available spectra. Columns (9) and (10) give the weighted mean velocity for each star and their uncertainties. Finally, notes for the individual stars are given in column (11). Table 4 contains the information for the doubtful stars, and Table 5 contains a similar list of nonmembers.

Twenty-six of the members are flagged as variables for various reasons. For 15 of these, multiple radial velocity measurements show them to be radial velocity variables. This is defined as having probabilities of less than $1 \%$ that their $\chi^{2}$ values are consistent with no variation. Eight stars, including two of the velocity variables, are identified in the lists of Rees (1992) or Tucholke et al. (1996) with variable stars from Nassau (1938) or Sawyer Hogg (1973). Most of these are RR Lyrae variables.

A further group have been flagged due to the velocity "jitter" seen in the brightest giants (Gunn \& Griffin 1979). Of the 11 giants in Figure 3 in the clump with $M<12.8$ and $M-T_{2}>1.55,8$ have multiple observations, and 6 of these are velocity variables. If we include an additional $0.8 \mathrm{~km} \mathrm{~s}^{-1}$ velocity uncertainty (Gunn $\&$ Griffin 1979) to allow for this jitter, then the velocities are consistent. We have removed these 11 stars from consideration for our velocity dispersion analysis.

\footnotetext{
${ }^{3}$ Stars identified by "Bu III-nn" are those listed in Table III of that paper. All others are from Table II.
}

TABLE 3

Data for Cluster Members

\begin{tabular}{|c|c|c|c|c|c|c|c|c|c|c|}
\hline $\begin{array}{l}\text { R.A. (J2000.0) } \\
\text { (1) }\end{array}$ & $\begin{array}{l}\text { Decl. (J2000.0) } \\
\text { (2) }\end{array}$ & $\begin{array}{l}\text { ID } \\
\text { (3) }\end{array}$ & $\begin{array}{c}r \\
(\operatorname{arcmin}) \\
(4)\end{array}$ & $\begin{array}{l}T_{2} \\
(5)\end{array}$ & $\begin{array}{l}M-T_{2} \\
\quad(6)\end{array}$ & $\begin{array}{l}C-M \\
\quad(7)\end{array}$ & $\begin{array}{l}N_{v}{ }^{\mathrm{a}} \\
(8)\end{array}$ & $\begin{array}{c}v \\
\left(\mathrm{~km} \mathrm{~s}^{-1}\right) \\
(9)\end{array}$ & $\begin{array}{c}\epsilon_{v} \\
\left(\mathrm{~km} \mathrm{~s}^{-1}\right) \\
(10)\end{array}$ & $\begin{array}{c}\text { Notes }^{\mathrm{b}} \text { and Other Names } \\
\text { (11) }\end{array}$ \\
\hline 171619.65 & +431426.0 & 1891 & 10.65 & 15.44 & 1.03 & 0.56 & 3 & -113.08 & 0.87 & $\mathrm{~T}$ \\
\hline $17 \quad 1622.24$ & +425804.9 & 516 & 13.01 & 13.62 & 1.09 & 0.75 & 13 & -119.02 & 0.09 & $\mathrm{~T}$ \\
\hline 171624.16 & $+4307 \quad 12.4$ & VII-3 & 7.88 & 15.45 & 1.06 & 0.53 & 2 & -125.16 & 0.52 & $\mathrm{~T}$ \\
\hline $17 \quad 1624.38$ & +431145.5 & 1924 & 8.55 & 15.47 & 1.02 & 0.49 & 4 & -118.05 & 0.44 & $\mathrm{~T}$ \\
\hline 171627.68 & +430855.1 & 2343 & 7.21 & 15.79 & 1.02 & 0.54 & 3 & -117.24 & 0.45 & \\
\hline 171629.83 & +430604.3 & VII-8 & 7.11 & 14.94 & 1.10 & 0.58 & 4 & -117.28 & 0.29 & $\mathrm{~T}$ \\
\hline 171629.99 & +430245.3 & 1995 & 8.68 & 15.53 & 0.96 & 0.55 & 4 & -124.87 & 0.57 & $\mathrm{~T}$ \\
\hline 171631.13 & +4304 46.5 & 3917 & 7.39 & 16.77 & 0.93 & 0.47 & 2 & -123.97 & 1.30 & \\
\hline 171633.36 & +43 1021.8 & 1936 & 6.51 & 15.48 & 1.03 & 0.54 & 3 & -124.71 & 0.54 & $\mathrm{~T}$ \\
\hline $17 \quad 1633.89$ & +430704.0 & 3180 & 6.15 & 16.37 & 0.96 & 0.50 & 3 & -119.18 & 0.72 & \\
\hline
\end{tabular}

Notes.-Units of right ascension are hours, minutes, and seconds, and units of declination are degrees, arcminutes, and arcseconds. Table 3 is published in its entirety in the electronic edition of the Astronomical Journal. A portion is shown here for guidance regarding its form and content.

" Number of observations. Where there is only one observation, the configuration code from Table 2 is given. For stars with configuration " $Z$," the velocity is based on the sum of all available spectra.

${ }^{\mathrm{b}} \mathrm{R}$, has a proper motion in Rees (1992); T, has a proper motion in Tucholke et al. (1996); v, variable in our data; V, known to be variable from another source; $\mathrm{j}$, velocities likely affected by jitter; $\mathrm{p}$, photometry is from a lower quality source. 
TABLE 4

Data for Doubtful Cluster Members

\begin{tabular}{|c|c|c|c|c|c|c|c|c|c|c|}
\hline R.A. (J2000.0) & Decl. (J2000.0) & ID & $\begin{array}{c}r \\
(\operatorname{arcmin})\end{array}$ & $T_{2}$ & $M-T_{2}$ & $C-M$ & $N_{v}^{\mathrm{a}}$ & $\begin{array}{c}v \\
\left(\mathrm{~km} \mathrm{~s}^{-1}\right)\end{array}$ & $\begin{array}{c}\epsilon_{v} \\
\left(\mathrm{~km} \mathrm{~s}^{-1}\right)\end{array}$ & Notes $^{\mathrm{b}}$ and Other Names \\
\hline 171559.02 & +430953.3 & 2171 & 12.52 & 15.67 & 0.88 & 0.55 & 5 & -89.33 & 0.39 & To \\
\hline 171619.64 & +431403.8 & 2919 & 10.44 & 16.20 & 0.91 & 0.49 & 3 & -100.89 & 1.06 & To \\
\hline 171732.80 & +431200.4 & 3448 & 6.05 & 16.52 & 1.07 & 0.62 & 3 & -94.31 & 0.88 & o \\
\hline 171812.18 & +425504.0 & 30 & 17.74 & 10.26 & 2.03 & 1.70 & $\mathrm{~J} 1$ & -108.45 & 1.12 & Tpo \\
\hline 171629.11 & +430944.9 & VI-7 & 7.09 & 12.66 & 1.03 & 0.65 & $\mathrm{~m} 1$ & -139.57 & 0.31 & RTc, ZNG-4 \\
\hline 171707.91 & +4307 12.2 & R644 & 1.00 & 14.03 & 1.09 & 1.06 & 3 & -121.84 & 0.92 & $\mathrm{Rh}$ \\
\hline 171839.80 & +430610.5 & 1016 & 17.05 & 14.91 & 0.70 & 0.70 & $\mathrm{~J} 2$ & -85.97 & 0.87 & $\mathrm{Th}$ \\
\hline
\end{tabular}

NotE.- Units of right ascension are hours, minutes, and seconds, and units of declination are degrees, arcminutes, and arcseconds.

a Number of observations. Where there is only one observation, the configuration code from Table 2 is given.

b $\mathrm{R}$, has a proper motion in Rees (1992); T, has a proper motion in Tucholke et al. (1996); o, outlying velocity; c, odd colors; h, high $h_{1}$ value; p, photometry is from a lower quality source.

In addition to the four discrepant stars, we also include in Table 4 three additional stars. The stars 1016 and R644 have accordant velocities but slightly high $h_{1}$ values and have thus been rejected from the membership list. On the other hand, star 1016 has an $81 \%$ probability of membership according to Tucholke et al. (1996) and R644 a 99\% probability of membership according to Rees (1992). It is probable that they really are members, but we have excluded them from the analysis for consistency. Finally, the star VI-7, which is the star at $7^{\prime}$ with a velocity of $-140 \mathrm{~km} \mathrm{~s}^{-1}$, has been rejected based on its peculiar DDO colors in Norris \& Zinn (1977).

The final result is that we have 306 stars we consider to be members and 7 stars that are doubtful members. We show the velocities of these stars against their radial position in Figure 7. The open circles indicate the 26 probable velocity variables mentioned above, while the crosses indicate the doubtful members. The median error for these velocities is $0.35 \mathrm{~km} \mathrm{~s}^{-1}$, with $92 \%$ having errors less than $1 \mathrm{~km} \mathrm{~s}^{-1}$.

Our sample of 306 stars includes 34 of the 35 stars for which Soderberg et al. (1999) have reported velocities. Comparison of our velocities with theirs indicates a very similar mean velocity for the stars in their sample. However, the individual velocity differences from the mean show a systematic deviation between the two studies, viz., their values for this difference are about $75 \%$ of what we find. Their template was constructed from an average of all their bright giants and includes strong interstellar $\mathrm{Na} \mathrm{D}$ lines as well (C. A. Pilachowski 2005, private communication). To the extent that a stellar spectrum is dominated by these interstellar lines, its cross-correlation velocity will be pulled toward the template velocity, i.e., to the cluster mean. Thus, the measured crosscorrelation velocity will be a weighted average of the cluster mean and the true velocity of the star. Since our spectral region does not contain strong interstellar lines, our velocities are likely to be more reliable.

\section{BAYESIAN ANALYSIS}

\subsection{General Principles}

As in Paper I, we derive the velocity dispersion profile from our velocities using Bayesian principles (Sivia 1996; Jaynes 2003; MacKay 2003). Our method here differs from and improves on that used in Paper I. The improvements are procedural and conceptual, as is discussed below.

Our approach is to consider a set of specified, parametric models for the velocity dispersion profile. These do not exhaust the full range of possible profiles, but they do provide a plausible starting set. We then infer the posterior probability distributions for the model parameters for each model on the basis of our observed velocities. We also calculate the relative likelihoods of the various classes of models with respect to one another. This allows us to identify the model best supported by the data.

Generically, if the vector $\boldsymbol{p}$ represents the parameters for a model or hypothesis $H$ and $D$ represents the available data, then,

TABLE 5

DATA FOR NONMEMBERS

\begin{tabular}{|c|c|c|c|c|c|c|c|c|c|}
\hline R.A. (J2000.0) & Decl. (J2000.0) & ID & $T_{2}$ & $M-T_{2}$ & $C-M$ & $N_{v}^{\mathrm{a}}$ & $\begin{array}{c}v \\
\left(\mathrm{~km} \mathrm{~s}^{-1}\right)\end{array}$ & $\begin{array}{c}\epsilon_{v} \\
\left(\mathrm{~km} \mathrm{~s}^{-1}\right)\end{array}$ & Notes $^{\mathrm{b}}$ and Other Names \\
\hline 171416.43 & +430940.8 & 40 & 10.86 & 1.42 & $\ldots$ & $\mathrm{J} 3 \mathrm{a}$ & -65.95 & 2.35 & $\mathrm{p}$ \\
\hline 171424.38 & +425039.0 & 594 & 13.42 & 1.36 & $\ldots$ & 2 & -89.90 & 0.44 & $\mathrm{p}$ \\
\hline 171424.44 & +425545.8 & 487 & 13.70 & 0.90 & 0.47 & $\mathrm{~J} 3 \mathrm{a}$ & -47.57 & 2.27 & $\mathrm{p}$ \\
\hline 171427.31 & +424450.4 & 389 & 13.11 & 1.24 & $\ldots$ & $\mathrm{J} 4$ & -3.30 & 1.53 & $\mathrm{p}$ \\
\hline 171430.21 & +430326.5 & 447 & 13.43 & 0.95 & 0.68 & $\mathrm{~J} 1$ & -2.41 & 0.76 & $\mathrm{p}$ \\
\hline 171432.91 & +4301 10.6 & 739 & 13.90 & 1.20 & 0.90 & 2 & -95.67 & 0.96 & p \\
\hline
\end{tabular}

NoтеS.- Units of right ascension are hours, minutes, and seconds, and units of declination are degrees, arcminutes, and arcseconds. Table 5 is published in its entirety in the electronic edition of the Astronomical Journal. A portion is shown here for guidance regarding its form and content.

a Number of observations. Where there is only one observation, the configuration code from Table 2 is given. For stars with configuration "Z," the velocity is based on the sum of all available spectra.

${ }^{b} \mathrm{R}$, has a proper motion in Rees (1992) and probability of membership $\geq 90 \%$; r, has a proper motion in Rees (1992) and probability of membership <90\%; $\mathrm{T}$, has a proper motion in Tucholke et al. (1996) and probability of membership $\geq 90 \%$; $\mathrm{t}$, has a proper motion in Tucholke et al. (1996) and probability of membership $<90 \%$; v, variable; $p$, photometry is from a lower quality source. 


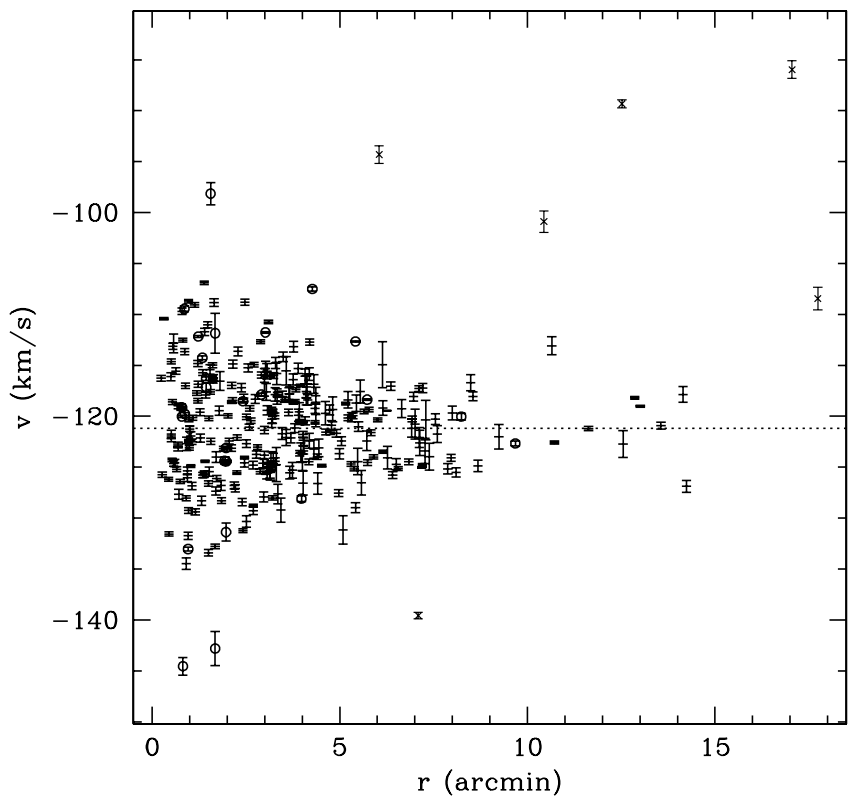

FIG. 7.- Velocities vs. radial distance for the members of M92. Circles represent mean velocities of the variables. Note the existence of stars with large velocities at large radii. The line indicates the mean velocity. The crosses represent the doubtful members. (The seventh doubtful member is in the clump near the mean velocity at $1^{\prime}$.)

given background information $I$, Bayes's theorem states that the posterior probability for $\boldsymbol{p}$ is given by

$$
p(\boldsymbol{p} \mid D H I)=\frac{p(D \mid \boldsymbol{p} H I) p(\boldsymbol{p} \mid H I)}{p(D \mid H I)} .
$$

The quantity $p(D \mid \boldsymbol{p} H I)=L(\boldsymbol{p})$ is the likelihood, which, for the present problem, we take to be

$$
L(\boldsymbol{p})=(2 \pi)^{-N / 2} \prod_{i=1}^{N} k_{i}^{1 / 2} \exp \left[-\frac{1}{2} \sum_{i=1}^{N}\left(v_{i}-\bar{v}\right)^{2} k_{i}\right],
$$

where, if $\epsilon_{i}$ is the uncertainty in velocity $v_{i}$ and $\sigma\left(r_{i}\right)$ is the dispersion appropriate to a star at radius $r_{i}$,

$$
k_{i} \equiv\left[\epsilon_{i}^{2}+\sigma\left(r_{i}\right)^{2}\right]^{-1} .
$$

The connection with maximum likelihood methods is discussed below.

The components of the parameter vector are the mean velocity, $\bar{v}$, and the various parameters required to define $\sigma(r)$. The mean velocity is a nuisance parameter in terms of inferring the velocity dispersion profile and can be removed by marginalizing over it.

The prior probability for $\boldsymbol{p}$ is given by $p(\boldsymbol{p} \mid H I)$. For the most part the parameters for the models we use are logically independent, so $p(\boldsymbol{p} \mid H I)$ will factor. The denominator, $p(D \mid H I)=$ $\int p(D \mid \boldsymbol{p} H I) p(\boldsymbol{p} \mid H I) d \boldsymbol{p}$, gives the overall probability of the data given a hypothesis that is variously termed the "evidence" or the "model likelihood." For a particular model this is just a normalization factor, but application of Bayes's theorem once again gives $p(H \mid D I) \propto p(D \mid H I) p(H \mid I)$ for the posterior probability of model $H$. When we wish to compare two models, $H_{1}$ and $H_{2}$, we can do this by taking the ratio of their posterior probabilities:

$$
\frac{p\left(H_{1} \mid D I\right)}{p\left(H_{2} \mid D I\right)}=\frac{p\left(D \mid H_{1} I\right)}{p\left(D \mid H_{2} I\right)} \frac{p\left(H_{1} \mid I\right)}{p\left(H_{2} \mid I\right)} .
$$

The first factor on the right-hand side is the likelihood ratio using the values of $p(D \mid H I)$ we just computed. The second factor is the ratio of the prior probabilities for the two models. Our procedure in Paper I had the shortcoming that instead of calculating $p(D \mid H I)$, we compared the maxima in $p(\boldsymbol{p} \mid D H I)$. This compares only the best set of parameters for each model. The proper thing to consider is the relative merits of the models for all combinations of parameters. Comparing the probabilities of the best sets of parameters favors a model having a narrow range of parameters that fit the data particularly well over one that has a broader range of parameters that fit the data somewhat less well, whereas the opposite ought to be the case. The issue is the choice of the best model class, not simply the best set of parameters.

In this we differ from maximum likelihood methods. The latter can be derived from the Bayesian method in the limit where the prior probabilities are constant. The objective is then to find the maximum, i.e., the mode, of the likelihood function and to report the function based on this set of parameters as "the velocity dispersion profile," in much the same way as we did in Paper I. The width of the likelihood function can be used to give uncertainties for the parameters, but this method has no way of assessing the relative merits of various possible descriptions of the data.

At the same time, it is often difficult to fully illustrate the complexity of the posterior probability function. For situations with a large number of parameters, Markov-chain Monte Carlo methods are practical but can take a long time to generate sufficient samples to ensure that the posterior is being properly sampled. For problems with relatively few parameters, direct calculation of the probability is a practical proposition. Consequently, in this paper we compute the joint probabilities $p(\boldsymbol{p} \mid D H I)$ directly on a multidimensional grid. The highest dimensional grid we use here has five dimensions, but the number depends on the model, as discussed in the next section. For models with radial binning, the parameter space naturally factors into smaller, tractable subspaces. In each dimension we used a grid of 50 points concentrated on the region of significant probability, which is, consequently, well resolved. We then extract the information in this function in several ways to highlight not only its maximum, but also certain aspects of its shape.

To this end we calculate the marginal probabilities, $p\left(p_{i} \mid D H I\right)$, of the each of the parameters, $p_{i}$, by integrating $p(\boldsymbol{p} \mid D H I)$ over all the other parameters in the problem. Numerically, we present our results in terms of the moments and mode of these marginal distributions. For each parameter we give the mean defined as the expectation value

$$
\left\langle p_{i}\right\rangle \equiv \int p_{i} p\left(p_{i} \mid D H I\right) d p_{i}
$$

and the standard deviation

$$
\epsilon_{p_{i}}=\sqrt{\left\langle p_{i}^{2}\right\rangle-\left\langle p_{i}\right\rangle^{2}}
$$

We also estimate the mode of the smooth marginal distributions by constructing a parabolic fit to the mode and the two bracketing points on the function grid. Deviations between the mode and the mean highlight asymmetries in $p\left(p_{i} \mid D H I\right)$. In such cases we 
use the following notation to indicate both these values: $[x]=$ $\langle x\rangle(x) \pm \epsilon_{x}$, where the value in parentheses is the mode. Otherwise, we just give the mean and $1 \sigma$ standard deviation as usual, although this should not be taken to necessarily indicate that the marginal distribution is Gaussian.

It should be noted that the modes of the marginal distributions correspond to the overall mode of $p(\boldsymbol{p} \mid D H I)$ only if the joint probability is symmetric. In practice, there are often significant correlations between parameters as highlighted by covariances calculated from joint probabilities of pairs of parameters. In order to highlight the range of parameters permitted by $p(\boldsymbol{p} \mid D H I)$, we give two graphical representations of our results in addition to the values mentioned in the previous paragraph. First, we show the marginal probability distributions for the best-fitting models. We also calculate $p(\sigma \mid r D H I)$ by integrating $p(\boldsymbol{p} \mid D H I)$ over regions of constant $\sigma(r)$. From these we show the mean value and uncertainty as defined by equations (5) and (6), as well as the mode. Again, the difference between the mode and mean highlight asymmetries in $p(\sigma \mid r D H I)$. In practice, these curves will also deviate from those based on the mean or modal parameters for the model, again because of asymmetries in the overall probability distribution. For ease of graphical comparison with later models, we tabulate some of the points on these curves. We wish to emphasize that the errors on these points are by no means independent. Comparisons between future models and these data are best undertaken with the original velocities, not with these curves. In light of these new, improved procedures, we also reanalyze the M15 data from Paper I.

\subsection{The Models}

In accord with the discussion in Drukier et al. (2003) highlighting the underlying difficulties in inferring global properties from a set of individual velocities, the velocity dispersion profiles to be derived below depend on the following assumptions. We assume that the velocities can be perfectly described by Gaussian distributions with a common mean and a dispersion that can vary with radius. Any higher order moments are ignored in our analysis. We assume that the uncertainties derived above for our radial velocities are accurate and precise and that the errors in the measured velocities are distributed as Gaussians with zero mean and standard deviation equal to these uncertainties. We assume that there are no nonmembers present in our sample. We do not exclude the possibility of there being unbound stars. Indeed, the use of a Gaussian implicitly assumes that the extreme tail is populated by unbound stars. We also assume that all the stars in our final sample are single stars with the same mass. It is possible that there are unidentified velocity variables, including binaries, in our sample. These would serve to inflate the dispersion. These provisos are included in the background information $I$. A model that could be considered is one in which the distribution of the velocities is consistent with the observed errors. Such a model, however, is strongly rejected with respect to all others, indicating that we detect the intrinsic variance in the cluster velocities. We now list the models we consider in this paper. This list is neither exclusive nor exhaustive, but it does cover likely variations. ${ }^{4}$

Model B.-The stars are sorted by distance from the cluster center and divided into bins. The velocity dispersion is taken to

\footnotetext{
${ }^{4}$ This is not to preclude other, potentially interesting models. The King (1966) model has often been used to describe globular clusters. With its energy cutoff, however, it explicitly cannot account for escaping stars, which would then have to be accounted for separately. Such two-component models, as discussed at the end of $\S 3.2$, are beyond the scope of this paper. Similarly, we do not investigate the models that include non-Newtonian effects (Scarpa et al. 2003, 2006).
}

be constant in each bin. This is the traditional approach, which we augment with our Bayesian estimation of the velocity dispersion. The parameters are the mean velocity of the sample $\bar{v}$, the number of bins $M$, and the set of dispersions $\left\{\sigma_{j}\right\}, j=$ $1, \ldots, M$. The choice of binning leads to various subtypes of this model class. Bins can be arbitrary, or divided roughly equally in terms of number of stars per bin or in terms of the radial range covered. An alternative is to let the numbers of stars per bin or, equivalently, the radial ranges be an additional set of $M-1 \mathrm{pa}-$ rameters, $r_{i}, i=1, \ldots, M-1$.

Model P.-The velocity dispersion profile has the form of a power law $\sigma(r)=\sigma_{1} r^{\alpha}$. The parameters of this model are $\bar{v} ; \sigma_{1}$, the velocity dispersion at $r=1^{\prime}$; and $\alpha$.

Model C.-The velocity dispersion profile has the form of a power law with a core,

$$
\sigma(r)=\sigma_{1}\left[\frac{1+\left(r / r_{0}\right)^{2}}{1+r_{0}^{-2}}\right]^{\alpha / 2}
$$

The parameters are the same as in model $P$, with the addition of the scale radius $r_{0}$ of the core in arcminutes. Note that, as in model $P, \sigma_{1}$ is normalized to be the value of the dispersion at $r=1^{\prime}$. This removes the correlation between $r_{0}$ and $\sigma_{1}$ that otherwise would be present. Model $C$ is identical to model $P$ in the limit $r_{0} \rightarrow 0$ and so is a more general case. For large $r, \sigma \propto r^{\alpha}$.

Model PB.-This model is a hybrid with a power-law inner section and an outer section in which the velocity dispersion is constant. This model is motivated by our discovery in Paper I that the velocity dispersion appears to increase in the outer part of M15. It seems sensible then to consider that these stars may not share the velocity dispersion properties of the rest of the stars in the cluster. The parameters here are the same as in model $P$, with the addition of the radius $r_{1}$ dividing the two populations and the dispersion, $\sigma_{\text {out }}$, for these outer stars.

Model $C B$.- This model is the same as model $P B$, but for the cored power law. It has the parameters of model $C$, plus $r_{1}$ and $\sigma_{\text {out }}$.

In both model $P B$ and model $C B$ the assumption is that there are distinct radial regions containing distinct populations of stars. The inner population consists of the stars bound to the cluster, while the outer population consists of those stars that deviate from the cluster distribution because of tidal perturbations, ejection from the cluster core, or other, unknown reasons. A more realistic model would be one in which the two populations are intermingled with additional parameters, including the membership probabilities for the two populations, describing the composite distribution. Similarly, the possibility of undiscovered binaries could be included. These are a more complicated proposition to implement than the models presented here but may be required to fully understand the data. A description of a similar approach can be found in $\S 21.5$ of Jaynes (2003).

\subsection{Priors}

We now consider the prior probabilities for the various parameters. The mean velocity is common to all the models, and we take $p(\bar{v} \mid H I)$ to be uniform. Its normalization will drop out of all model comparisons, so we can safely use a flat, improper prior. For the various dispersions, the correct prior is the Jeffreys prior (Sivia 1996; Jaynes 2003) $p(\sigma \mid H I) \propto \sigma^{-1}$. This is equivalent to a flat prior in $\log (\sigma)$. We need to set sensible bounds, $\sigma_{-}<$ $\sigma<\sigma_{+}$, on this in order to make it normalizable. We have used $\sigma_{-}=0.3 \mathrm{~km} \mathrm{~s}^{-1}$, since this is the median error on our velocities, and smaller dispersions are likely to be meaningless. For the 
TABLE 6

Permitted Ranges for Parameters

\begin{tabular}{|c|c|c|c|c|c|}
\hline \multirow[b]{2}{*}{ Parameter } & \multirow[b]{2}{*}{ Models } & \multicolumn{2}{|c|}{ M92 } & \multicolumn{2}{|c|}{ M15 } \\
\hline & & Lower & Upper & Lower & Upper \\
\hline $\bar{v}\left(\mathrm{~km} \mathrm{~s}^{-1}\right) \ldots \ldots \ldots$ & All & -122.58 & -119.58 & -108.5 & -105 \\
\hline$\sigma_{1}\left(\mathrm{~km} \mathrm{~s}^{-1}\right) \ldots \ldots$. & All & 0.3 & 12 & 0.3 & 20 \\
\hline$\alpha \ldots \ldots+\cdots$ & $P, P B, C, C B$ & -2.5 & 0 & -2.5 & 0 \\
\hline$r_{0}(\operatorname{arcmin}) \ldots \ldots \ldots$ & $C, C B$ & 0.3 & 8 & 0.3 & 3 \\
\hline$r_{1}(\operatorname{arcmin}) \ldots \ldots \ldots$ & $P B, C B$ & 8 & 14 & 7.5 & 15 \\
\hline$\sigma_{\text {out }}\left(\mathrm{km} \mathrm{s}^{-1}\right) \ldots \ldots$ & $P B, C B$ & 0.3 & 10 & 0.3 & 10 \\
\hline
\end{tabular}

upper limit we use values appropriate to each cluster. For the models using the power-law index $\alpha$, we have taken $p(\alpha \mid H I)$ to be uniform over $-2.5<\alpha<0$, since the velocity dispersion should be a decreasing function of radius, but not too strongly. The lower limit is set by consideration of the range of physically sensible values for $\alpha$.

For models $C$ and $C B$, since we interpret $r_{0}$ as a scale parameter, the correct prior is the Jeffreys prior. It is meaningless to speak of a core radius outside the radial range of the data, so the limits on $r_{0}$ are taken with this in mind.

Finally, for models $P B$ and $C B$, as well as variants of model $B$ with varying bin sizes, we take $p\left(r_{i} \mid H I\right)$ to be uniform over the appropriate radial range. In this study we consider two different sorts of binnings. The first is a standard arrangement, with the stars divided into bins of equal number. The second is to set a minimum number of stars per bin and then scan through all possible combinations of break radii in order to find the optimal bin size. We denote models with these two binnings as $B_{N=M}$ and $B_{S=M}$, respectively, where $M$ is the number of bins. A scanning approach similar to the $B_{S}$ models is taken to the break radius $r_{1}$ in models $P B$ and $C B$, and, again, the prior is uniform over a specified radial range.

Note that for models in class $B$ with $M>2$, the prior probabilities on the positions of the bin boundaries are not independent, since they are sequential and we require each bin to contain a minimum number of stars. For $M=3$, for example, $p\left(r_{1} r_{2} \mid B_{S=3} I\right)=$ $p\left(r_{1} \mid B_{S=3} I\right) p\left(r_{2} \mid r_{1} B_{S=3} I\right)$ follows from the product rule of probabilities, with similar expressions for additional bin boundaries. The limits on the various parameters for the M92 and M15 data sets are given in Table 6 .

The selection of priors for the models themselves is a somewhat more difficult proposition. In some cases we may indeed have pertinent prior information. For M15, for example, the surface density and surface brightness profiles are power law in form to within $2^{\prime \prime}$ of the center of the cluster (Sosin \& King 1997). Inspection of the velocity dispersion profile in Figure 9 of Gerssen et al. (2002) suggests that the break in the slope of the velocity dispersion occurs somewhat farther out, at around $1^{\prime}$ to within a factor of 2. This difference is to be expected. If, for example, we consider the single-mass $W_{0}=9$ King (1966) model shown in Figure 4-11 of Binney \& Tremaine (1987), we can see that the line-of-sight velocity dispersion drops to half its central value at about $30 r_{c}$. We could consider this an effective core radius for the velocity dispersion profile, $r_{c, v}$. Thus, it would not be unreasonable to expect to see some evidence for flattening within our data sample. If the core radius in the velocity dispersion is unresolved, it may be difficult to differentiate between a true core and the situation we see in example PL+F discussed in $\S 3.5$. Taking this into account, $p\left(P \mid I_{\mathrm{M} 15}\right) / p\left(C \mid I_{\mathrm{M} 15}\right) \sim 5$ would not be unreasonable for the two options of a power law (model $P$ ) and a power law with core (model $C$ ). This expresses our relative ambivalence with respect to the two models but a desire for some stronger evidence of flattening before we are prepared to believe in it. Like any prior, it is a statement of how strongly we hold any given proposition.

For M92, our preference is for model $C$ over model $P$, since the cluster shows a clear core radius of $14^{\prime \prime}$ in the density data (Trager et al. 1995). We would then expect $r_{c, v} \sim 7^{\prime}$. In terms of the parameterization of model $C, r_{0}=r_{c, v} /\left(4^{-1 / \alpha}-1\right)^{1 / 2}$. For $\alpha<-0.25, r_{0}>1^{\prime}$ and is well within our data sample. Thus, we expect to see clear evidence for a core in the velocity data. In principle, this argument can lead to a stronger joint prior on $r_{0}$ and $\alpha$ than the relatively uninformative priors proposed above, but we refrain from using such a joint prior here. Instead, we take $p\left(C \mid I_{\mathrm{M} 92}\right) / p\left(P \mid I_{\mathrm{M} 92}\right)=100$, expressing our strong prior expectation of model $C$.

In the absence of suitable prior information, the same considerations that lead to the numerical values above suggest we employ the "device of imaginary results" of Good (1950). Before doing the analysis of the results, we ask ourselves how much better the likelihood of one model over another would have to be for us to consider the two models equally probable. The inverse of this ratio is then the desired prior. If, for example, the possible models for a set of globular cluster data are a model with or a model without a central black hole of unknown mass, and, being skeptical of the proposition, we would need the likelihood in support of a model with a black hole to be 50 times that without, then the proper prior ratio is $p(B \mid D I) / p(\bar{B} \mid D I)=1 / 50$, where $B$ is the proposition that the cluster contains a black hole and $\bar{B}$ is its negation.

The case of binned models is interesting in this regard. No one actually thinks of a globular cluster as being stratified in the way implied by binning. The main reason to use such a model is to look for features in the profile not described by the simpler parametric models, such as $P, C$, etc. Increasing the number of bins allows for finer detail in the resulting profile but also decreases the precision of the measurement of the dispersions. The Bayesian formalism naturally takes this effect into account. A model with more bins is penalized by the additional priors attached to additional parameters. If, nonetheless, the total probability is maximized for a particular set of bins, this is a way to optimize the choice. Such optimization is, of course, subject to the prior on the number of bins, but we take that to be uniform up to some maximum number of bins. Our objective with this class of models is to look for the most probable binning in case there is some obvious feature not captured by the other models under consideration. Nonetheless, given the lack of physical motivation for a binned model, we set $p(B \mid I)$ to be 0.02 times the prior probability of the preferred model of $P$ or $C$ as appropriate for M92 and M15. This probability is intended to apply to the entire collection of binned models, reflecting the flexibility the observer has in choosing the binning. In the subset of models with variablewidth bins we require a minimum of 20 stars per bin for $M=2$, 3 , or 4 . (The calculation becomes very long for higher values of $M$ in this case.) Applying the same limit to bins with an equal number of stars per bin permits all models with $M \leq 14$ for the M92 data and $M \leq 10$ for the M15 data set. Dividing $p(B \mid I)$ equally between the 17 cases for M92 and 13 cases for M15 gives relative prior probabilities of $1.2 \times 10^{-3}$ and $1.5 \times 10^{-3}$ per binned model for the two clusters. Finally, we consider the odds to be even on whether the outer zones in models $P B$ and $C B$ exist.

At this point we invite the reader to decide what prior ratios are appropriate for the various models we describe in $\S 3.2$ in the cases of M92 and M15. Below, we present the results in such a 
TABLE 7

Results for Mock Data Sets

\begin{tabular}{|c|c|c|c|c|c|}
\hline \multirow[b]{2}{*}{ Model } & \multicolumn{5}{|c|}{$\log [p($ model $\mid D I) / p($ best model $\mid D I)]$} \\
\hline & PL & CPL & $\mathrm{PL}+\mathrm{F}$ & $\mathrm{D}$ & S \\
\hline$\ldots \ldots \ldots \ldots$ & 0.0 & -1.2 & -7.3 & -5.8 & -2.1 \\
\hline$\ldots \ldots \ldots \ldots \ldots$ & -1.7 & 0.0 & -8.8 & -4.0 & -1.8 \\
\hline$P B \ldots \ldots \ldots \ldots \ldots \ldots$ & -0.5 & -1.7 & -0.04 & -6.6 & -3.7 \\
\hline$C B$ & -2.3 & -0.3 & 0.0 & -5.1 & -3.4 \\
\hline$B_{N}(1-20) \ldots \ldots$ & -9.5 & -3.0 & -5.7 & -1.5 & 0.1 \\
\hline$B_{N}$ (best) $\ldots \ldots \ldots$ & $-9.9(11)$ & $-3.3(7)$ & $-6.1(7)$ & $-1.5(5)$ & $0.0(1)$ \\
\hline$B_{S}(2-4) \ldots \ldots \ldots$ & -10.5 & -4.3 & -6.9 & 0.1 & 0.0 \\
\hline$B_{S}$ (best) ........ & $-10.5(4)$ & $-4.3(3)$ & $-6.9(4)$ & $0.0(3)$ & $-0.1(2)$ \\
\hline
\end{tabular}

way that the posterior odds can be recalculated for other choices of prior ratios.

\subsection{Implementation Details}

The posterior probability distribution for each model was calculated on an $N$-dimensional grid, where $N$ is the number of parameters in the model. The marginal probability for each parameter was calculated from the values on this grid by numerical integration over all the other parameters. Along the way, the twodimensional marginalizations were examined and used to calculate the covariances between pairs of parameters. To increase the efficiency of the calculations, we first conducted a pilot study using a coarse grid covering the full ranges of the priors. This was used to locate the region of nontrivial probability in each parameter, which was then covered by a finer grid in our final calculation. The marginal probability distributions were saved. We also calculated the first and second moments of these to give the expectation value and variance of the distributions. In cases in which the posterior probability distribution is close to a Gaussian, these are close to the mean and variance of that Gaussian.

In the cases in which one or more break radii are themselves parameters, the above calculation was done for each set of break radii, and the total probability is naturally the marginal probability of that set of radii. The posterior probability distributions of the break radii are highly non-Gaussian, and rather than specifying the rather unenlightening moments, we use the mode as the typical value. The other parameters were marginalized over the break radii.

\subsection{Examples}

We now proceed to use some mock data sets to demonstrate the use of our methods. In all cases the radial distributions and velocity uncertainties are those of our M92 data. For each mock data set we used our Bayesian procedures to estimate the parameters for all the models discussed. For the binned models we tried 1-20 equal-number bins and 2-6 scanned bins, i.e., the cases with one to five break points. For the scanned bins a minimum bin size of 40 stars was used. We present our results in Table 7. For each of the mock data sets we give the log of the likelihood ratio of each model with respect to the best model for that data set. For each of the binning schemes the total probability presented is the sum of the total probabilities for all binnings. We also give the results for the best value of $M$ in each binning scheme. In all cases the mean velocity of the mock data sets was consistent with the input value. Although we have dropped the units for simplicity, they should be clear from the context. We discuss each mock data set in turn.
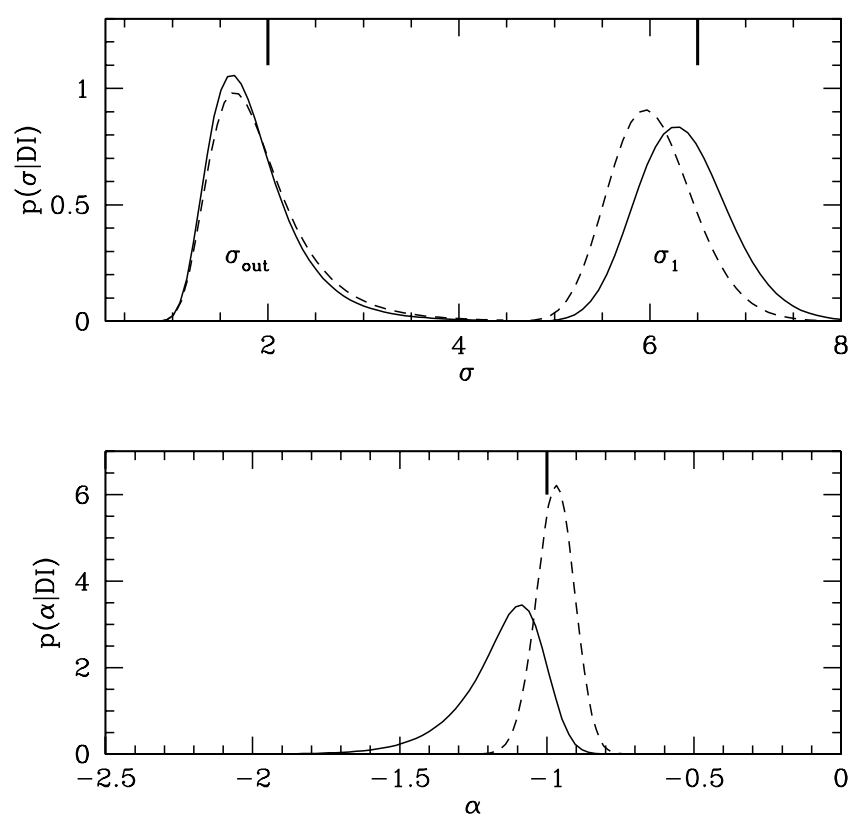

FIG. 8.-Probability density distributions for the velocity dispersions (top) and power-law index (bottom) for the mock data set PL+F. Solid lines, model $C B$; dashed lines, model $P B$. The thick vertical lines are at the input values of the three parameters.

$P L$.-A power law with $\sigma_{1}=6.5$ and $\alpha=-1$. The best model is $P$ with $\left\langle\sigma_{1}\right\rangle=6.0 \pm 0.4$ and $\langle\alpha\rangle=-1.05 \pm 0.06$. Model $C$ is 50 times worse, and, since the best core radius is at the lower limit of the prior, this supports the primacy of model $P$ in this case. The only comparable model is $P B$, in which the $\sigma_{1}$ and $\alpha$ probability distributions are nearly identical. In the outer region, $p\left(\sigma_{\text {out }} \mid D I\right)$ is strongly peaked at the lower limit of the allowed range. In other words, the results for model $P B$ are effectively identical to those of model $P$, but the model is penalized by its additional parameters. The steep gradient makes the constant dispersion within each of the bins in model $B$ a very poor representation of the data.

CPL.-A cored power law with $\sigma_{1}=6.5, \alpha=-1$, and $r_{0}=1$. The best model is $C$ with $\left\langle\sigma_{1}\right\rangle=6.2 \pm 0.5,\langle\alpha\rangle=-1.0 \pm 0.1$, and $\left\langle r_{0}\right\rangle=1.1 \pm 0.4$. The next best model is $C B$, but the situation here is the same as in the previous case. The parameters in the inner region are the same, and the fit is penalized by the existence of the additional parameters.

$P L+F$.- A power law with $\sigma_{1}=6.5$ and $\alpha=-1$ inside a radius of 8 , but beyond this the dispersion is flat at 2 . The most probable model is $C B$, with $P B$ running a very close second. The core radius probability distribution is strongly peaked at the lower limit of the permissible range (0.3), giving only an upper limit. This indicates some flattening of the dispersion profile toward small radii, but the core is not resolved. This is easily understood in terms of the spatial distribution of the stars. In the innermost sampled region, only a small fraction of the stars have measured velocities. The probability of any of these stars having a high velocity is small, so the velocity dispersion appears to increase less quickly at the inner edge of the data. Thus, we can see hints of a core radius, even when the model underlying the mock data contains no such flattening. The estimated values of $\sigma_{1}$ and $\alpha$ are similar in the two models but are less well-defined in the more general model $C B$, as seen in Figure 8. As is also shown, the two models agree on the dispersion in the outer region. The asymmetry in the probability density distribution for $\sigma_{\text {out }}$ is typical of dispersions measured from a small number of stars. For 


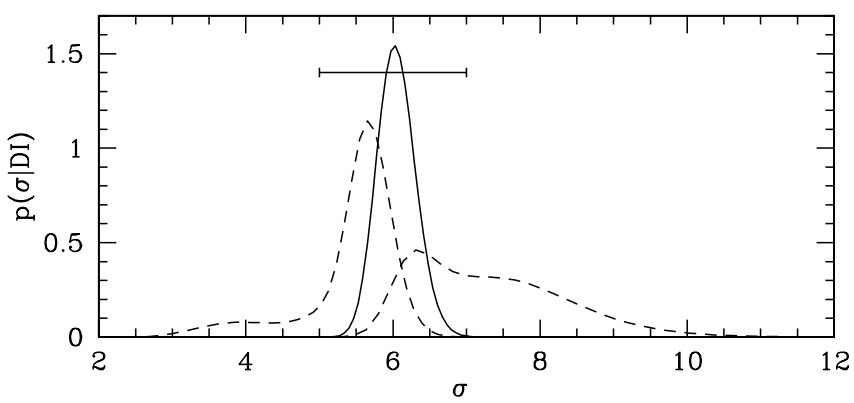

FIG. 9.- Probability density distributions for the velocity dispersion for mock data set $S$. Solid line, single dispersion model; dashed lines, two-dispersion model. The line at the top represents the range of input dispersions for this data set.

model $\mathrm{CB}\left\langle\sigma_{1}\right\rangle=6.4 \pm 0.5,[\alpha]=-1.2(-1.1) \pm 0.1,\left[r_{0}\right]=$ $0.6(0.3) \pm 0.3$, and $\left[\sigma_{\text {out }}\right]=1.9(1.6) \pm 0.5$, while for model $P B$ $\left\langle\sigma_{1}\right\rangle=6.0 \pm 0.4,\langle\alpha\rangle=-0.97 \pm 0.06$, and $\left[\sigma_{\text {out }}\right]=1.9(1.7) \pm$ 0.5 . There is a minor disagreement as to the location of the boundary between the two regions based on which side the three points lying between $8.3^{\prime}$ and $10^{\prime}$ are placed.

D.-A flat distribution with $\sigma=6.5$, except between $1.5^{\prime}$ and $3.5^{\prime}$, where the dispersion is double. This is admittedly unrealistic but is designed to show the flexibility of the procedure. The best model is a binned one with two adjustable boundaries giving three bins. The modal boundaries lie at $1.53^{\prime}$ and $3.56^{\prime}$, and the velocity dispersions in the three bins are $\left\langle\sigma_{1}\right\rangle=7.2 \pm 0.7$, $\left\langle\sigma_{2}\right\rangle=13.2 \pm 1.0$, and $\left\langle\sigma_{3}\right\rangle=7.4 \pm 0.5$, with the bins containing 65,91 , and 118 stars in order of radius. The next best model is one with three adjustable bin boundaries, but the final result is degenerate in the sense that the outer two boundaries have the same mode. This model is 7 times less probable than the best model. The other models are at best $10^{4}$ times less probable and must be rejected for any sensible prior.

$S$.-A velocity dispersion given by $\sigma(r)=5.0+2.0 \sin (r \pi / 14)$. This mock data set is designed to show what happens when none of the models are suitable to the data. In this case the most probable model is simply a flat distribution with $\left\langle\sigma_{1}\right\rangle=6.1 \pm 0.3$. Nearly as likely is a two-zone model with a modal bin boundary at $6^{\prime}$. In this model $\left\langle\sigma_{1}\right\rangle=5.6 \pm 0.4$ and $\left[\sigma_{2}\right]=7.3(6.5) \pm 0.9$. As shown in Figure 9 the probability distributions for this latter model are quite non-Gaussian, with the probability distributions for both dispersions showing hints of bimodality. Results like these for real data would indicate a great cause for concern.

We now turn to our velocities in M92 and M15 and analyze them in the same way.

\section{KINEMATIC RESULTS}

\section{1. $M 92$}

We give the results of our Bayesian analysis of the new M92 data in Table 8 . The likelihood of our five model classes relative to model $C B$ is given in the second column of the table. With the priors in the third column - based on the discussion in $\S 3.3$ - the posterior probability of the model classes is given in the fourth column. Model $C B$ is the most likely, but model $C$ is only a factor of 2 less probable, so we consider them together. Models $P$ and $P B$ are an order of magnitude less likely than mode $C B$, confirming the existence of a core, and with our prior information they are up to $10^{3}$ times less probable.

Of the $B$-class models, the model with two equal-sized bins is significantly more likely than any of the others. It is interesting to note that the model with two adjustable bins comes up with much the same result, shuffling eight stars from the inner to the outer
TABLE 8

RESULTS FOR M92

\begin{tabular}{|c|c|c|c|c|c|c|}
\hline Model & $\log$ & {$\left[\frac{p(D \mid H I)}{p(D \mid C B I)}\right]^{\mathrm{a}}$} & $\log$ & {$\left[\frac{p(H \mid I)}{p(C B \mid I)}\right]^{\mathrm{b}}$} & $\log$ & {$\left[\frac{p(H \mid D I)}{p(C B \mid D I)}\right]$} \\
\hline$P$ & & -0.5 & & -2.0 & & -2.5 \\
\hline 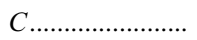 & & -0.2 & & 0.0 & & -0.2 \\
\hline$P B \ldots \ldots \ldots \ldots \ldots \ldots$ & & -0.9 & & -2.0 & & -2.9 \\
\hline$C B$ & & 0.0 & & 0.0 & & 0.0 \\
\hline$B$ & & 0.1 & & -1.7 & & -1.6 \\
\hline$B_{N=2} \ldots \ldots \ldots \ldots$ & & 0.0 & & -2.9 & & -2.9 \\
\hline$B_{N=3} \ldots \ldots \ldots \ldots \ldots \ldots$ & & -1.4 & & -2.9 & & -4.3 \\
\hline$B_{N=4} \ldots \ldots \ldots \ldots \ldots \ldots$ & & -1.5 & & -2.9 & & -4.4 \\
\hline$B_{N=5} \ldots \ldots \ldots \ldots \ldots$ & & -2.1 & & -2.9 & & -5.0 \\
\hline$B_{N=6} \ldots \ldots \ldots \ldots$ & & -3.2 & & -2.9 & & -6.1 \\
\hline$B_{S=2} \ldots \ldots \ldots \ldots$ & & -0.7 & & -2.9 & & -3.6 \\
\hline$B_{S=3} \ldots \ldots \ldots \ldots \ldots \ldots$ & & -1.1 & & -2.9 & & -4.0 \\
\hline$B_{S=4} \ldots \ldots \ldots \ldots \ldots$ & & -1.8 & & -2.9 & & -4.7 \\
\hline
\end{tabular}

${ }^{\text {a }}$ Log relative likelihood.

${ }^{\mathrm{b}}$ Log relative prior probability.

${ }^{c}$ Log relative posterior probability.

bin, at the cost of a lower likelihood due to the prior on the position of the bin boundary. The $B_{N=2}$ model has dispersions of $\left\langle\sigma_{1}\right\rangle=5.8 \pm 0.4$ and $\left\langle\sigma_{2}\right\rangle=3.5 \pm 0.2 \mathrm{~km} \mathrm{~s}^{-1}$. At the bottom of Table 8 we give the likelihoods relative to model $C B$ for the most likely binned models. Increasing the number of bins does not improve the match to the data. Given our prior, we reject the binned models, individually and as a class.

The probability density distributions for the various parameters in models $C B$ and $C$ are shown in Figure 10. The probability distributions for the mean velocity and $\sigma_{1}$ are consistent for both models and give $\langle\bar{v}\rangle=-121.2 \pm 0.3$ and $\left\langle\sigma_{1}\right\rangle=6.3 \pm 0.5 \mathrm{~km} \mathrm{~s}^{-1}$ for model $C$ and $\langle\bar{v}\rangle=-121.1 \pm 0.3$ and $\left\langle\sigma_{1}\right\rangle=6.2 \pm 0.5 \mathrm{~km} \mathrm{~s}^{-1}$ for model $C B$. They differ in the parameters giving the shape of
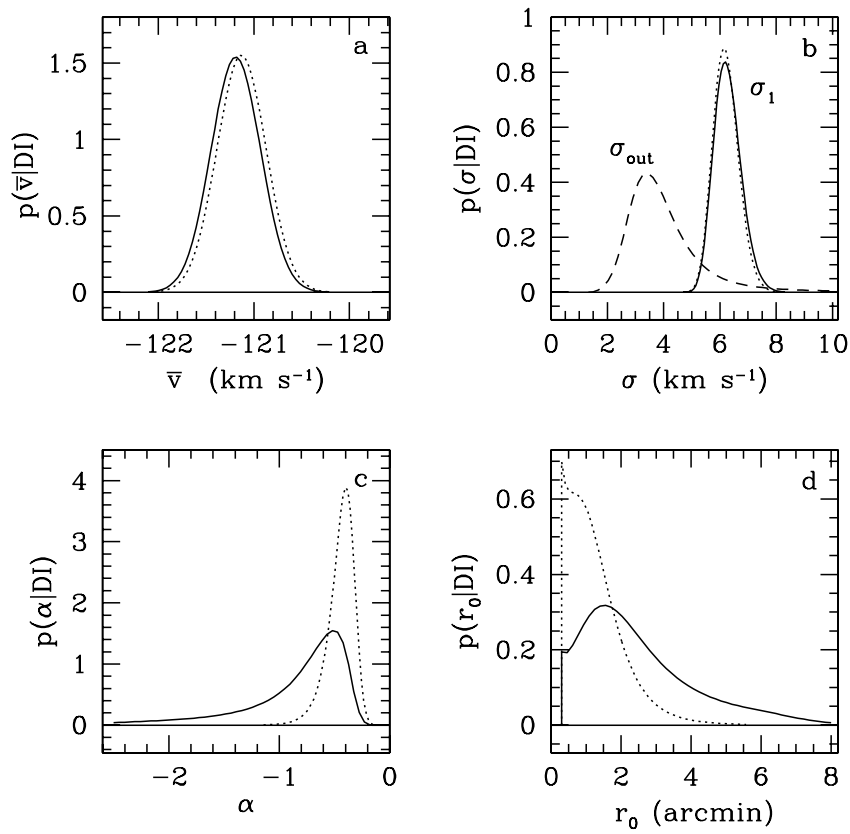

FIG. 10.-Probability density distributions for models $C B$ (solid lines) and $C$ (dotted lines) of the full M92 data set. (a) Mean velocity. (b) Velocity dispersion at $1^{\prime}$. The dashed line shows the distribution for $\sigma_{\text {out }}$ in the $C B$ model. The long tail to higher velocity is typical of dispersion distributions based on only a few stars. (c) Power-law slope. (d) Core radius. 


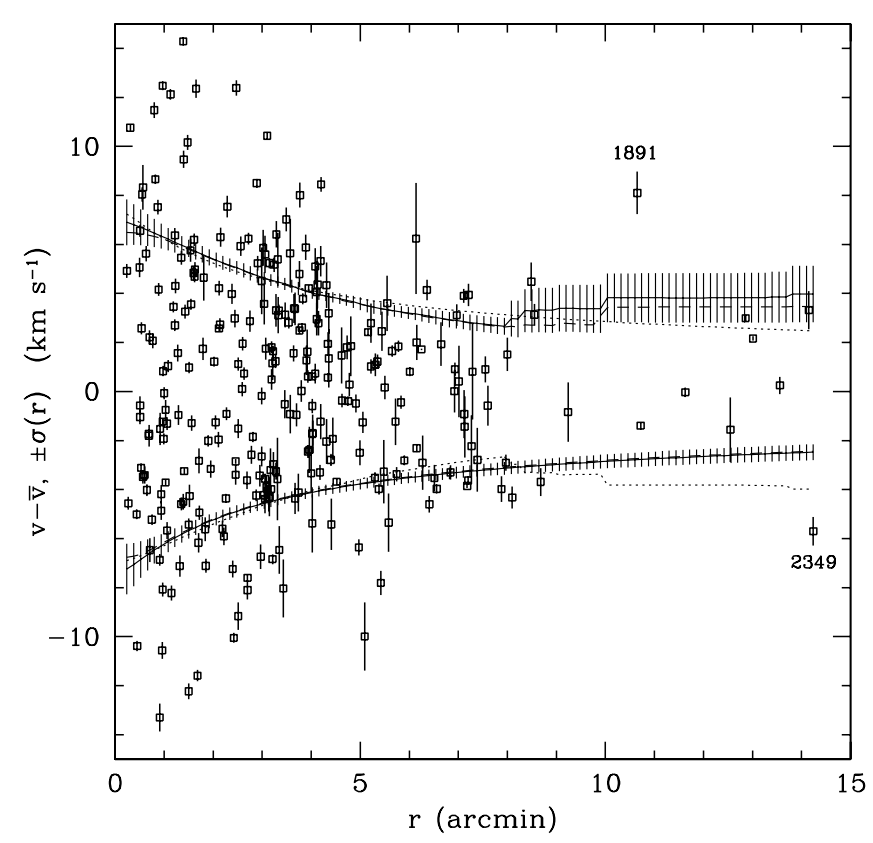

FIG. 11. - Velocity dispersion profiles for our M92 data plotted with the velocities. The solid curves represent the mean of $p(\sigma \mid r D I)$, while the shading indicates the $1 \sigma$ errors based on this distribution. The dashed curves indicate the modal results as discussed in the text. The upper curves are for model $C B$, while the lower curves are for model $C$. The mean curves for each distribution are reflected about zero and plotted as dotted curves to ease comparison. The two stars discussed in the text are labeled.

the velocity dispersion profile. For model $C B,[\alpha]=-0.8(-0.5) \pm$ 0.4 and $\left[r_{0}\right]=2.5^{\prime}\left(1.5^{\prime}\right) \pm 1.6^{\prime}$. As can be seen in Figure 10, the probability distributions for these quantities are broad and asymmetric. In addition, they are anticorrelated; shallow profiles with narrow cores work equally as well as steep profiles with broad cores. The modal value for $r_{1}$ is at $10^{\prime}$, and it appears that the stars inside this radius can only weakly constrain the shape of the curve. This can be seen graphically in Figure 11. (Fiducial points for the displayed M92 profiles are given in Table 9.) The upper shaded region shows the $1 \sigma$ spread in $p\left(\sigma \mid r D C B I_{\mathrm{M} 92}\right)$. Inside $8^{\prime}$, a range of velocity profiles are possible. Outside this radius, the profile is affected by the possible break radius and independent velocity dispersion. (This can be seen in the steplike increase in the outer part of model $C B$. For such models, one of the parameters is the boundary $r_{1}$, and this parameter has a fairly wide range of values with nontrivial probability. Consequently, this part of the profile can be either inside or outside $r_{1}$, and the curve reflects this ambiguity.) For these data and model $C B,\left[\sigma_{\text {out }}\right]=4.0(3.4) \pm 1.3 \mathrm{~km} \mathrm{~s}^{-1}$. The asymmetry in the probability distribution (see Fig. 10b) is typical of cases in which there are few stars available to constrain the value of the dispersion.

For model $C,\langle\alpha\rangle=-0.4 \pm 0.1$ and $\left[r_{0}\right]=1.3^{\prime}\left(0.3^{\prime}\right) \pm 0.8^{\prime}$. The former is well constrained, but the latter has its mode at the lower limit of its permitted range. This indicates that there is some flattening to the profile, but the core appears smaller than would be expected. The profile is shown as the lower shaded region in Figure 11. Model $C$ is strongly constrained by the three outer stars with large deviations from the mean velocity. In order to account for these velocities, a relatively shallow yet coreless profile is favored. Model $C B$ puts these stars in the outer zone, removing their influence on the parameters of the inner profile, but the velocities of the remaining stars can be fit by a large range of profiles. Thus, model $C B$ is preferred.
TABLE 9

Mean Points for M92

\begin{tabular}{|c|c|c|c|c|}
\hline \multirow[b]{2}{*}{$\begin{array}{c}r \\
(\operatorname{arcmin})\end{array}$} & \multicolumn{2}{|c|}{ Model $C$} & \multicolumn{2}{|c|}{ Model $C B$} \\
\hline & $\begin{array}{c}\langle\sigma(r)\rangle \\
\left(\mathrm{km} \mathrm{s}^{-1}\right)\end{array}$ & $\begin{array}{c}\epsilon_{\sigma(r)} \\
\left(\mathrm{km} \mathrm{s}^{-1}\right)\end{array}$ & $\begin{array}{c}\langle\sigma(r)\rangle \\
\left(\mathrm{km} \mathrm{s}^{-1}\right)\end{array}$ & $\begin{array}{c}\epsilon_{\sigma(r)} \\
\left(\mathrm{km} \mathrm{s}^{-1}\right)\end{array}$ \\
\hline $0.24 \ldots \ldots \ldots \ldots \ldots$ & 7.25 & 1.06 & 6.90 & 0.93 \\
\hline $0.80 \ldots \ldots \ldots \ldots \ldots$ & 6.54 & 0.57 & 6.46 & 0.56 \\
\hline 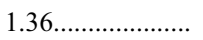 & 5.89 & 0.36 & 5.96 & 0.38 \\
\hline $1.92 \ldots \ldots \ldots \ldots \ldots$ & 5.35 & 0.29 & 5.48 & 0.31 \\
\hline $2.48 \ldots \ldots \ldots \ldots \ldots$ & 4.90 & 0.26 & 5.04 & 0.28 \\
\hline $3.04 \ldots \ldots \ldots \ldots \ldots$ & 4.54 & 0.24 & 4.64 & 0.26 \\
\hline $3.60 \ldots \ldots \ldots \ldots \ldots$ & 4.24 & 0.22 & 4.31 & 0.28 \\
\hline 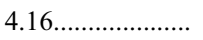 & 3.98 & 0.22 & 3.95 & 0.22 \\
\hline $4.72 \ldots \ldots \ldots \ldots \ldots$ & 3.76 & 0.21 & 3.73 & 0.23 \\
\hline $5.28 \ldots \ldots \ldots \ldots \ldots \ldots$ & 3.58 & 0.22 & 3.44 & 0.26 \\
\hline $5.84 \ldots \ldots \ldots \ldots \ldots \ldots . .$. & 3.41 & 0.22 & 3.25 & 0.24 \\
\hline 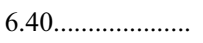 & 3.27 & 0.23 & 3.07 & 0.28 \\
\hline 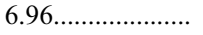 & 3.14 & 0.24 & 2.89 & 0.32 \\
\hline $7.52 \ldots \ldots \ldots \ldots \ldots$ & 3.03 & 0.25 & 2.75 & 0.33 \\
\hline $8.08 \ldots \ldots \ldots \ldots \ldots .$. & 2.93 & 0.26 & 2.97 & 0.72 \\
\hline $8.64 \ldots \ldots \ldots \ldots \ldots . . .$. & 2.84 & 0.26 & 3.32 & 0.89 \\
\hline 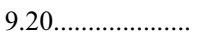 & 2.75 & 0.27 & 3.39 & 0.94 \\
\hline 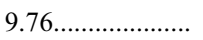 & 2.68 & 0.28 & 3.37 & 0.97 \\
\hline $10.32 \ldots \ldots \ldots \ldots$ & 2.61 & 0.29 & 3.82 & 0.98 \\
\hline 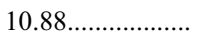 & 2.54 & 0.29 & 3.81 & 0.98 \\
\hline 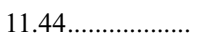 & 2.48 & 0.30 & 3.81 & 0.99 \\
\hline $12.00 \ldots \ldots \ldots \ldots \ldots$ & 2.43 & 0.31 & 3.81 & 0.99 \\
\hline $12.56 \ldots \ldots \ldots \ldots \ldots \ldots$ & 2.38 & 0.31 & 3.81 & 0.99 \\
\hline $13.12 \ldots \ldots \ldots \ldots \ldots \ldots \ldots$ & 2.33 & 0.32 & 3.82 & 1.00 \\
\hline $13.68 \ldots \ldots \ldots \ldots \ldots . .$. & 2.28 & 0.32 & 3.84 & 1.02 \\
\hline 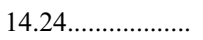 & 2.24 & 0.32 & 3.97 & 1.14 \\
\hline
\end{tabular}

The most deviant velocity is that of star 1891 in our list, lying at $10.7^{\prime}$. Under some circumstances one might be tempted to reject it from the cluster sample outright. This happens to be the westernmost star in our sample. It is fairly faint but lies on the red giant branch in the CMD. It has three relatively low-quality velocity measurements, but these agree within their respective uncertainties. Tucholke et al. (1996) give the star only an $8 \%$ probability of membership, but this is not the only star with a low Tucholke et al. (1996) probability that satisfies our criteria for membership. A multicomponent model such as that described at the end of $\S 3.2$ would directly incorporate borderline cases such as this by assigning some probability to their membership. For the present, we just go to the other extreme and redo the analysis without star 1891.

The results for $\bar{v}$ and $\sigma_{1}$ are the same with or without this star. For model $C B\left[\sigma_{\text {out }}\right]=3.9(2.8) \pm 1.7 \mathrm{~km} \mathrm{~s}^{-1}$, the large skew and uncertainty reflecting the fact that this distribution is derived from only the two outermost stars. For both model $C B$ and model $C$ the probability distributions for $\alpha$ and $r_{0}$ are somewhat broader and shifted with respect to the distributions in Figure 10. For model $C B[\alpha]=-0.9(-0.6) \pm 0.4$ and $\left[r_{0}\right]=2.8^{\prime}\left(1.8^{\prime}\right) \pm 1.6^{\prime}$. As in the previous case, $\sigma_{1}$ and $\alpha$ are strongly correlated with $r_{0}$. For model $C\left\langle\sigma_{1}\right\rangle=6.3 \pm 0.5 \mathrm{~km} \mathrm{~s}^{-1}$ and $\langle\alpha\rangle=-0.5 \pm 0.2$. There is now a peak to the $r_{0}$ distribution, and $\left[r_{0}\right]=1.5^{\prime}\left(1.1^{\prime}\right) \pm 0.9^{\prime}$.

From a comparison of the results of model $C$ probability distributions for all possible subsamples obtainable by dropping a single star, that obtained by dropping star 1891 stands out. Dropping this star gives a significantly larger increase in the model probability than is the case for any other star. The only comparable subsample is the one dropping the outermost star (2349). In only these two cases do the model parameters differ from those 


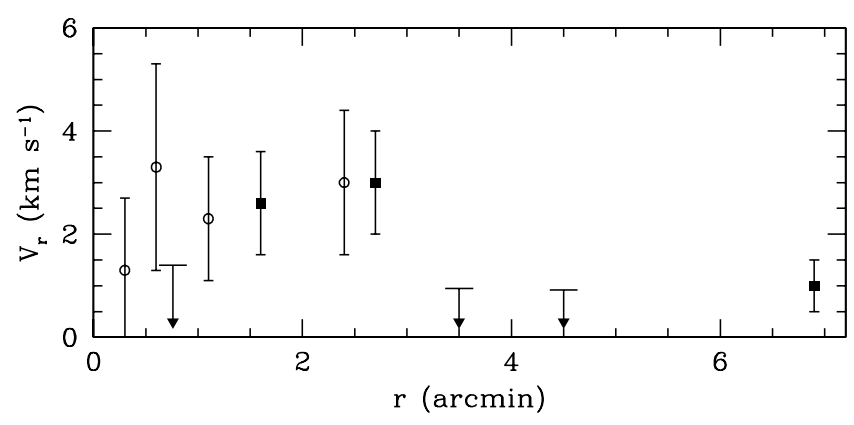

FIG. 12.- Rotation amplitude for M92 as a function of radius. The first, fourth, and fifth symbols indicate $1 \sigma$ upper limits. The open symbols represent the bins with detected rotation from Lupton et al. (1985).

given by the full sample. And it is only in these two cases that the mode of $r_{0}$ is not at the lower limit of the permitted range. Given these results, it is possible that it is only these two stars that are anomalous, rather than all the stars beyond a certain radius. Such a suggestion constitutes a new model class, one in which a certain fraction of the stars are excluded, but we do not pursue this model here. We do note, however, that a $C$-class model without either of these stars gives $\langle\bar{v}\rangle=121.1 \pm 0.2 \mathrm{~km} \mathrm{~s}^{-1},\left\langle\sigma_{1}\right\rangle=$ $6.3 \pm 0.5 \mathrm{~km} \mathrm{~s}^{-1},\langle\alpha\rangle=-0.7 \pm 0.3$, and $\left[r_{0}\right]=2.2^{\prime}\left(1.7^{\prime}\right) \pm$ $1.2^{\prime}$. From a consideration of the various samples and models discussed above, it appears that $\sigma_{1}=6.3 \pm 0.5 \mathrm{~km} \mathrm{~s}^{-1}, \alpha=$ $-0.6 \pm 0.3$, and $r_{0}=2^{\prime} \pm 1^{\prime}$ is a reasonable description of the M92 velocity dispersion profile. For these values of $\alpha$ and $r_{0}$ we can calculate $r_{c, v}=6^{\prime}$, a value consistent with our expectations based on the density profile as discussed in $\S 3.3$.

From these data there is little evidence for a dominant population of stars in the outskirts of M92 with higher-than-expected velocities. We have, however, identified two stars that are highly inconsistent with the velocity distribution of the remaining stars even though their properties are otherwise consistent with cluster membership. We discuss the possible origin of these stars following a reevaluation of the M15 velocities.

\subsubsection{Rotation}

Although this paper is focused on the velocity dispersion profile, we would like to briefly follow up on the claim of Lupton et al. (1985) that the cluster is rotating. To that end, we have taken our preferred M92 model from the previous section, assumed it to be correct, and looked for sinusoidal rotational signals for six radial bins. We then subtracted off this signal and estimated new parameters for the profile model, iterating in this fashion until the values of the parameters stopped changing. The parameters for the velocity dispersion profile are consistent with those given above, except for $\sigma_{1}$, which drops to $6.0 \pm 0.5 \mathrm{~km} \mathrm{~s}^{-1}$. The rotation profile is shown in Figure 12 and is compared to that of Lupton et al. (1985). (We have used their value of $r_{c}=0.74 \mathrm{pc}$ and a distance of $8.2 \mathrm{kpc}$ to convert their abscissae to arcminutes.) A rotational signal is only seen in half the bins; for the others a $1 \sigma$ upper limit is shown. The position angle is consistent for the bins in which a signal is seen.

\section{2. $M 15$}

In Paper I we concluded that there was an indication that the velocity dispersion of M15 increased at large radii. In Table 10 we present our likelihoods and posterior probabilities in the same way as in Table 8. (Fiducial points for the displayed M15 profiles are given in Table 11.) In this case the most likely model is $C B$, but once our prior ratios are taken into account, the most prob-
TABLE 10

RESULTS FOR M15

\begin{tabular}{|c|c|c|c|c|c|c|}
\hline Model & $\log$ & {$\left[\frac{p(D \mid H I)}{p(D \mid C B I)}\right]^{\mathrm{a}}$} & $\log$ & {$\left[\frac{p(H \mid I)}{p(C B \mid I)}\right]^{\mathrm{b}}$} & $\log$ & {$\left[\frac{p(H \mid D I)}{p(C B \mid D I)}\right]$} \\
\hline$P \ldots \ldots \ldots \ldots$ & & -0.3 & & 0.0 & & -0.3 \\
\hline C................ & & -0.6 & & -0.7 & & -1.3 \\
\hline$P B \ldots \ldots \ldots$ & & -0.3 & & 0.0 & & -0.3 \\
\hline$C B \ldots \ldots \ldots$ & & 0.0 & & -0.7 & & -0.7 \\
\hline$B \ldots \ldots \ldots \ldots$ & & -0.9 & & -1.7 & & -2.6 \\
\hline$B_{N=2} \ldots \ldots$ & & -3.2 & & -2.9 & & -6.1 \\
\hline$B_{N=3} \ldots \ldots$ & & -0.9 & & -2.9 & & -3.8 \\
\hline$B_{N=5} \ldots \ldots$ & & -3.0 & & -2.9 & & -5.9 \\
\hline$B_{N=6} \ldots \ldots$ & & -3.4 & & -2.9 & & -6.3 \\
\hline$B_{N=7} \ldots \ldots$ & & -2.8 & & -2.9 & & -5.7 \\
\hline$B_{S=2} \ldots \ldots$ & & -1.9 & & -2.9 & & -4.8 \\
\hline$B_{S=3} \ldots \ldots \ldots$ & & -1.7 & & -2.9 & & -4.6 \\
\hline$B_{S=4} \ldots \ldots \ldots$ & & -1.9 & & -2.9 & & -4.8 \\
\hline
\end{tabular}

${ }^{\text {a }}$ Log relative likelihood.

${ }^{\mathrm{b}} \mathrm{Log}$ relative prior probability.

${ }^{c}$ Log relative posterior probability.

able models are $P$ and $P B$ by a small margin. The probability densities are plotted in Figure 13. None of the binned profiles are at all probable.

The higher likelihood of model $C B$ with respect to models $P B$ and $P$ lies in its ability to give a slightly flatter profile in the center. In this case the $r_{0}$ distribution is strongly peaked at its lower limit, indicating that whatever core there is in the velocity profile is unresolved. This is not unexpected since, as we discussed in $\S 3.3$, the velocity dispersion profile in M15 flattens at around $1^{\prime}$. For

TABLE 11

Mean Points for M15

\begin{tabular}{|c|c|c|c|c|}
\hline \multirow[b]{2}{*}{$\begin{array}{c}r \\
(\operatorname{arcmin})\end{array}$} & \multicolumn{2}{|c|}{ Model $P$} & \multicolumn{2}{|c|}{ Model $P B$} \\
\hline & $\begin{array}{c}\langle\sigma(r)\rangle \\
\left(\mathrm{km} \mathrm{s}^{-1}\right)\end{array}$ & $\begin{array}{c}\epsilon_{\sigma(r)} \\
\left(\mathrm{km} \mathrm{s}^{-1}\right)\end{array}$ & $\begin{array}{c}\langle\sigma(r)\rangle \\
\left(\mathrm{km} \mathrm{s}^{-1}\right)\end{array}$ & $\begin{array}{c}\epsilon_{\sigma(r)} \\
\left(\mathrm{km} \mathrm{s}^{-1}\right)\end{array}$ \\
\hline $0.08 \ldots \ldots \ldots \ldots \ldots \ldots$ & 20.85 & 4.60 & 28.99 & 8.38 \\
\hline $0.74 \ldots \ldots \ldots \ldots \ldots \ldots$ & 8.47 & 0.83 & 9.29 & 1.08 \\
\hline $1.41 \ldots \ldots \ldots \ldots \ldots \ldots$ & 6.59 & 0.46 & 6.80 & 0.51 \\
\hline $2.07 \ldots \ldots \ldots \ldots \ldots \ldots$ & 5.68 & 0.32 & 5.65 & 0.33 \\
\hline $2.73 \ldots \ldots \ldots \ldots \ldots \ldots$ & 5.10 & 0.26 & 4.96 & 0.26 \\
\hline $3.40 \ldots \ldots \ldots \ldots \ldots \ldots$ & 4.69 & 0.23 & 4.47 & 0.25 \\
\hline $4.06 \ldots \ldots \ldots \ldots \ldots \ldots$ & 4.38 & 0.22 & 4.12 & 0.25 \\
\hline $4.72 \ldots \ldots$ & 4.14 & 0.22 & 3.84 & 0.26 \\
\hline $5.39 \ldots \ldots \ldots \ldots \ldots \ldots$ & 3.94 & 0.23 & 3.61 & 0.27 \\
\hline 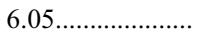 & 3.77 & 0.23 & 3.43 & 0.28 \\
\hline 6.71.................... & 3.63 & 0.23 & 3.27 & 0.28 \\
\hline $7.38 \ldots \ldots$ & 3.50 & 0.24 & 3.13 & 0.29 \\
\hline $8.04 \ldots \ldots \ldots \ldots \ldots \ldots$ & 3.39 & 0.24 & 3.05 & 0.33 \\
\hline $8.71 \ldots \ldots \ldots \ldots \ldots \ldots \ldots$ & 3.29 & 0.25 & 3.05 & 0.43 \\
\hline 9.37 ................. & 3.20 & 0.25 & 3.40 & 0.62 \\
\hline $10.03 \ldots \ldots \ldots \ldots \ldots$ & 3.12 & 0.25 & 3.44 & 0.65 \\
\hline $10.69 \ldots \ldots \ldots \ldots \ldots$ & 3.05 & 0.26 & 3.65 & 0.68 \\
\hline $11.36 \ldots \ldots \ldots \ldots \ldots \ldots$ & 2.98 & 0.26 & 3.77 & 0.69 \\
\hline $12.02 \ldots \ldots \ldots \ldots \ldots$ & 2.92 & 0.26 & 3.77 & 0.70 \\
\hline $12.68 \ldots \ldots \ldots \ldots \ldots \ldots$ & 2.86 & 0.26 & 3.79 & 0.70 \\
\hline $13.35 \ldots \ldots \ldots \ldots \ldots \ldots$ & 2.81 & 0.27 & 3.79 & 0.71 \\
\hline $14.01 \ldots \ldots \ldots \ldots \ldots \ldots$ & 2.76 & 0.27 & 3.79 & 0.72 \\
\hline $14.67 \ldots \ldots \ldots \ldots \ldots . . . . . .$. & 2.71 & 0.27 & 3.81 & 0.75 \\
\hline $15.34 \ldots \ldots \ldots \ldots \ldots$ & 2.67 & 0.27 & 3.81 & 0.75 \\
\hline $16.00 \ldots \ldots \ldots \ldots \ldots$ & 2.63 & 0.27 & 3.81 & 0.75 \\
\hline $16.66 \ldots \ldots \ldots \ldots \ldots$ & 2.59 & 0.27 & 3.81 & 0.75 \\
\hline
\end{tabular}



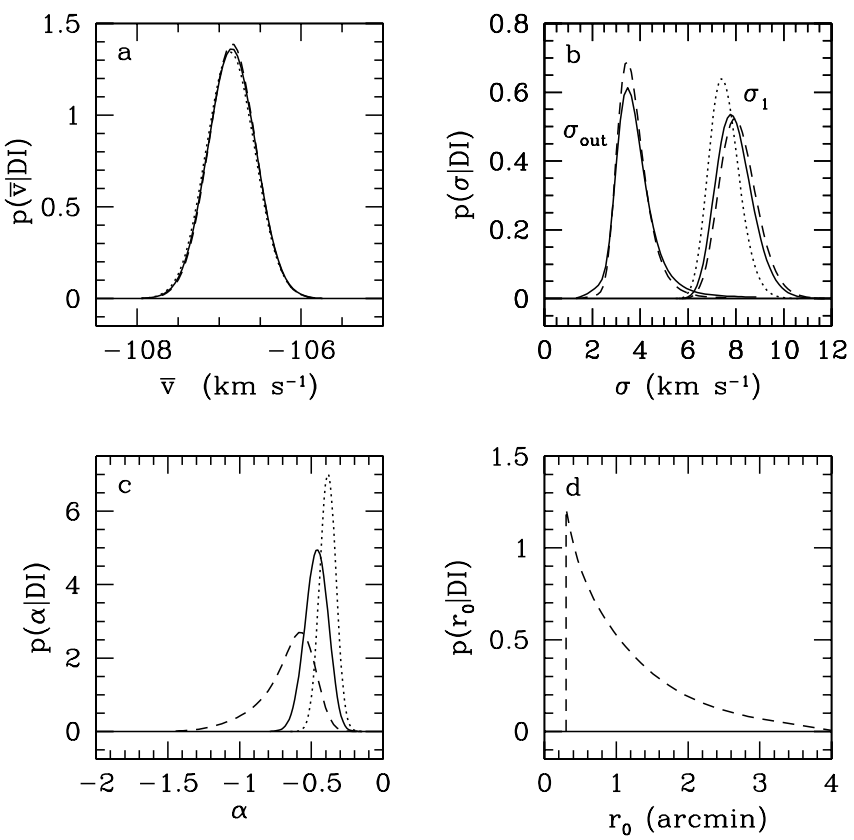

FIG. 13.- Probability density distributions for models $P$ (dotted lines), $P B$ (solid lines), and $C B$ (dashed lines) of the M15 data set. (a) Mean velocity. (b) Velocity dispersion at $1^{\prime}\left(\sigma_{1}\right)$ and in the outer region $\left(\sigma_{\text {out }}\right)$. The outer region contains more stars than in the comparable case in M92, so the distributions of $\sigma_{\text {out }}$ are less skewed. (c) Power-law slope. $(d)$ Core radius for model $C B$.

model $C B\left\langle\sigma_{1}\right\rangle=8.1 \pm 0.8 \mathrm{~km} \mathrm{~s}^{-1},\langle\alpha\rangle=-0.7 \pm 0.2,\left[r_{0}\right]=$ $1.1^{\prime}\left(0.3^{\prime}\right) \pm 0.7^{\prime}$, and $\left\langle\sigma_{\text {out }}\right\rangle=3.7 \pm 0.7 \mathrm{~km} \mathrm{~s}^{-1}$. Unlike M92, $\alpha$ and $r_{0}$ are uncorrelated here. The modal value for $r_{1}$ is $9^{\prime}$.

The profiles for models $P$ and $P B$ are shown in Figure 14. The two models differ in the way they accommodate the stars beyond $9^{\prime}$. The slope and scale of the power law are mainly set by the high-velocity stars of the inner cluster. For model $P B\left\langle\sigma_{1}\right\rangle=$ $8.0 \pm 0.8 \mathrm{~km} \mathrm{~s}^{-1},\langle\alpha\rangle=-0.47 \pm 0.08$, and $\left\langle\sigma_{\text {out }}\right\rangle=3.8 \pm$ $0.9 \mathrm{~km} \mathrm{~s}^{-1}$, with the modal value for $r_{1}$ at $9^{\prime}$. Model $P$ is constrained to a slightly shallower $\operatorname{drop}(\langle\alpha\rangle=-0.39 \pm 0.06)$ and a smaller central amplitude $\left(\left\langle\sigma_{1}\right\rangle=7.5 \pm 0.6 \mathrm{~km} \mathrm{~s}^{-1}\right)$. Nonetheless, the velocity dispersion profile at large radii for model $P$ is less than that of model $P B$. Model $P B$ fits the data somewhat better, but the improvement is balanced by the need for two extra parameters, and the result is that the two descriptions remain equally probable. On the one hand, the data are compatible with a single power law, but on the other, they do not exclude at any level the possibility that some fraction of the stars outside about $9^{\prime}$ have a somewhat higher velocity dispersion than that expected by extrapolating the most probable power law for the stars inside that radius.

\section{COMPARISON WITH $N$-BODY MODELS}

In Paper I we suggested that the apparent flattening or upturn in the velocity dispersion of M15 at large radii was due to the effect of the Galactic tidal field. Drukier et al. (1999), however, showed that during core collapse, a globular cluster ejects stars from the core. It is therefore worthwhile to consider the effects of unbound stars on the estimation of the velocity dispersion profile. While the seemingly systematic effect in M15 can be explained either way, the apparent high-velocity members seen in M92 might more naturally be explained by the ejection mechanism.

We have been carrying out a separate program to simulate the dynamic evolution of star clusters using GRAPE $N$-body

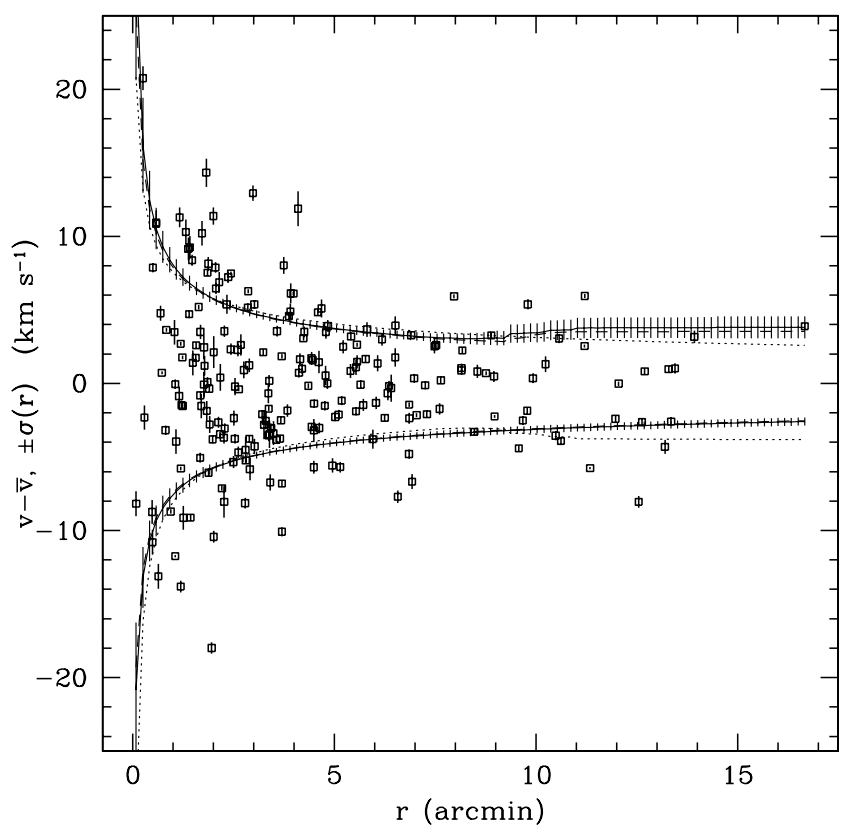

FIG. 14.- Same as Fig. 11, but for the M15 data from Paper I. The upper curve is for model $P B$ and the lower for model $P$.

supercomputers $^{5}$ at Indiana University. We have run series of isolated cluster models with identical stars to well beyond core collapse, as a benchmark for comparison with simulations that include tidal effects. Unlike our previous Fokker-Planck models of isolated clusters (Drukier et al. 1999), full $N$-body models allow the possibility of stars acquiring positive energies, i.e., exceeding the escape velocity. As noted by Johnston et al. (1999), the increasing dominance of the halo velocity distribution by escaping stars can produce a flattening of the halo velocity dispersion profile. We examine one such model here to illustrate the effects on the velocity dispersion profile of unbound stars expelled from the core.

For comparison with the M92 results, we used $N$-body data from a GRAPE-4 run with $N=8192$ identical point-mass stars. The initial state was a Plummer model with no primordial binaries. We used the NBODY4 code (Aarseth 1999) to evolve the model through core collapse and well into the postcollapse phase. Stellar escape occurred primarily as a result of cumulative energy increase from single-single scattering in the contracting core. This produced an isotropic stream of escaping stars. The escape rate increased as the model approached core collapse, with about $2.5 \%$ of the cluster mass lost to escape at the time of core collapse. As a compromise between M92 and M15, we selected a data snapshot from near the time of core collapse for analysis.

In order to compare this $N$-body model to the M92 data, we rescaled the $N$-body positions to give the model roughly the same limiting radius as M92. The velocities were scaled arbitrarily to give velocities with numerical values similar to the M92 values. We then selected stars from these projected lists to have roughly the same radial distribution as the M92 data. We did this in two ways. First, we selected our sample from only the bound stars. Then we reselected the sample in the same way, but this time we allowed unbound stars to enter each sample. Each of these unbound stars effectively replaced one bound star in the bound

\footnotetext{
${ }^{5}$ See Makino et al. (1997) for a description of the GRAPE hardware development program.
} 


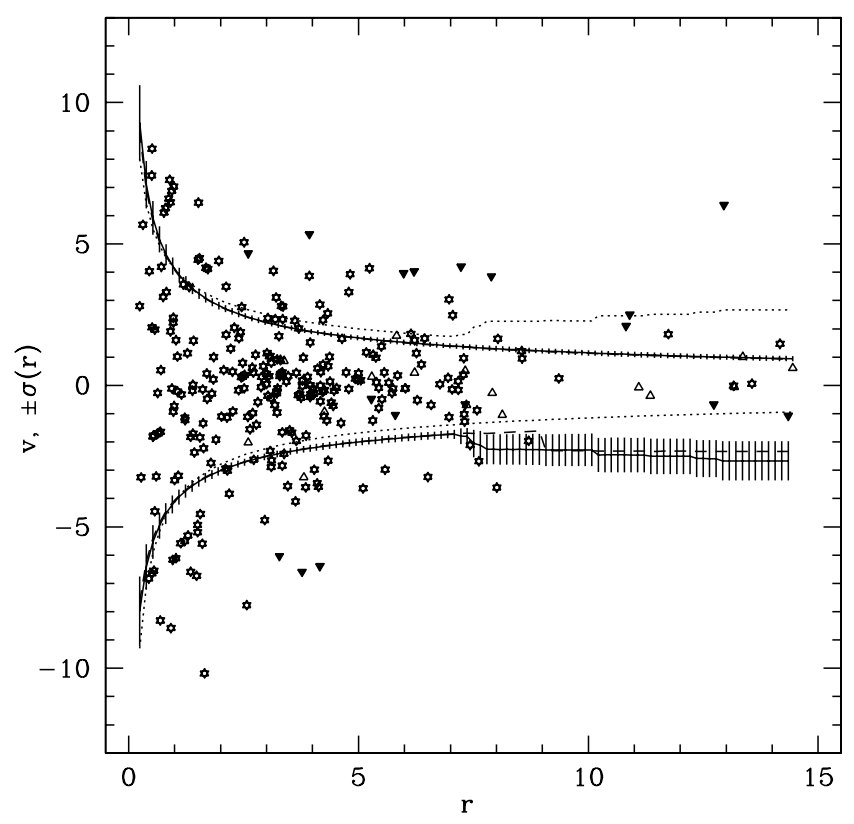

FIG. 15.- Stellar samples and most probable profiles for the $N$-body model. The six-pointed stars indicate stars selected for both the bound and unbound samples. The filled triangles represent unbound stars that replace the stars used only in the bound sample (open triangles). The curves are the same as in Fig. 11. The upper shading is for model $P$ for the bound sample. The lower shading is for model $P B$ for the unbound sample.

sample, so the total number of stars was maintained. In each case a single, obvious, strongly unbound "outlier" was rejected. As in $\S 3.5$, the units for the parameters should be obvious from the context.

We show the results for a typical projection in Figure 15. Stars common to both samples are shown as six-pointed stars. Filled triangles represent unbound stars in the second sample. They replace the bound stars from the first sample, shown as open triangles. The shaded regions and curves are the same as in Figure 11. The best model class for the bound samples is shown at positive velocities, while that for the unbound samples is shown at negative velocities. The dotted lines show the reflections of the mean curves about zero velocity for the purposes of comparison.

For the bound sample, models $P$ and $C$ are equally likely, but the $r_{0}$ distribution for the latter is peaked toward the center, supporting the slightly higher probability of model $P$. For model $P$ $\left\langle\sigma_{1}\right\rangle=4.1 \pm 0.3$ and $\langle\alpha\rangle=-0.56 \pm 0.06$. We show model $P$. For the unbound sample there are 17 unbound stars out of the remaining 279 . The most likely model is $B_{S=2}$ with $\left\langle\sigma_{1}\right\rangle=4.5 \pm$ 0.4 inside 1.76 and $\left\langle\sigma_{2}\right\rangle=2.2 \pm 0.1$ outside that radius. A factor of 3 less likely, but more probable on physical grounds, is model $P B$ (shown in Fig. 15), with the outermost nine stars in the outer zone for the modal value of $r_{1}=10$. Of these, five are indeed unbound. The outer dispersion is $\left[\sigma_{\text {out }}\right]=2.7(2.3) \pm 0.8$, but the difference between the mean and the mode indicates a strong skew to higher values, since there are few data points to constrain it. In the inner region $\left\langle\sigma_{1}\right\rangle=4.1 \pm 0.3$ and $\langle\alpha\rangle=$ $-0.44 \pm 0.06$. The central scale is the same as for the bound sample, but the slope of the power law is now somewhat flatter in order to take into account the velocities of the unbound stars at intermediate radii. Model $P$ is a factor of 9 less likely, with $\left\langle\sigma_{1}\right\rangle=3.9 \pm 0.3$ and $\langle\alpha\rangle=-0.36 \pm 0.05$. The unbound star at a radius of 12 affects the model in much the same way as star 1891 in M92. Removing this star provides the greatest improvement to model $P$ and gives $\left\langle\sigma_{1}\right\rangle=4.1 \pm 0.3$ and $\langle\alpha\rangle=$ $-0.43 \pm 0.05$, nearly identical to the previous model $P B$, where this star is beyond $r_{1}$. The effect of the other unbound stars remains, however.

From this comparison it appears that unbound stars can indeed modify the inferred velocity dispersion profile, generally giving a shallower slope than we find when only bound stars are used. Extreme outliers at large radii can be identified by the effect their removal from the sample has on the parameters and overall model probability. On the basis of this admittedly limited examination it would appear that the two rejected stars in M92 are likely to be unbound stars currently exiting the cluster.

M15, being in deep core collapse, should have more escaping stars and show larger effects, but the details depend on the radial distribution of the unbound stars. It is possible that the sample from Paper I contains some unbound stars at relatively small radii and, consequently, the velocity dispersion profile has been inflated in the inner region. The continuation to larger radii is consistent with the velocities in that region, perhaps disguising the effects of high-velocity stars there. In any case, the ejection of unbound stars from the cluster core is a stochastic process, and not all snapshots or projections will contain such stars or have the same radial distribution of unbound stars. What this example does demonstrate, however, is that sporadic star ejection from the core can produce an apparent increase in the velocity dispersion in the outer region. This is especially so considering that, going out from the core, the unbound stars form an increasing fraction of the cluster-associated stars.

\section{SUMMARY}

We have carried out an extensive investigation of the global radial velocity field in M92, using the WIYN telescope for both photometry and spectroscopy. We obtained high-accuracy (median error $0.35 \mathrm{~km} \mathrm{~s}^{-1}$ ) velocities for 306 probable cluster members, thereby greatly increasing the number of stars with measured radial velocities in this rich cluster, which has a very high-density yet resolved core. This new data set nicely complements that from our previous study of the collapsed-core cluster M15 (Paper I).

We selected likely cluster candidate members for spectroscopy by several photometric methods. The most efficient of these involved obtaining a large-area, three-band Washington photometry mosaic of M92 with WIYN. Using a photometric metallicity index, we were able to successfully identify cluster members with $\sim 70 \%$ efficiency within the projected cluster halo $\left(r>3^{\prime}\right)$, where the vast majority of stars are nonmembers.

We have introduced an improved Bayesian analysis scheme and applied it to both this data set and the M15 data set of Paper I. Of our models, the most probable one for the M92 data is that described by a core radius of about $2^{\prime}$, a velocity dispersion at $1^{\prime}$ of $6.3 \mathrm{~km} \mathrm{~s}^{-1}$, and an outer power-law slope of between -0.5 and -0.8 , depending on which, if any, of two outer high-velocity stars are included. It is these stars that appear to increase the dispersion in the outer part of the cluster. The M15 velocities can be described equally well by both a single power law with a slope of -0.5 and a velocity dispersion at $1^{\prime}$ of $8.0 \mathrm{~km} \mathrm{~s}^{-1}$ and by an inner power law with a slope of -0.4 and a scale of $7.5 \mathrm{~km} \mathrm{~s}^{-1}$ plus an outer region beyond $9^{\prime}$ with a dispersion of $4 \mathrm{~km} \mathrm{~s}^{-1}$. This region could be populated by some fraction of unbound stars. It is not unlikely that a few unbound stars are also present in the inner region of the cluster, particularly between $2^{\prime}$ and $5^{\prime}$. There is also some evidence that the slope of the power law flattens at the inner limit of our sample.

Our consideration of the velocity profiles in a GRAPE-based $N$-body model of an isolated cluster suggests that our two data sets may contain a number of escaping stars that were boosted to positive energies by the same internal relaxation processes that 
drive core collapse. Clearly, more data are needed to answer the questions of whether the velocity profiles do indeed flatten at large radii and to identify the physical mechanism behind this. For a realistic cluster in a tidal environment, the development of the halo must involve an interplay between internal two-body relaxation and external tidal influences.

Measures of mass segregation and velocity anisotropy may provide the best means to gauge the relative importance of twobody relaxation and tidal effects in determining the structure of cluster halos. Mass segregation is best investigated by very deep imaging. Andreuzzi et al. (2000) have used HST WFPC2 photometry to investigate mass segregation in M92. They find a significant change in the slope of the mass function between fields at 11 and 19 core radii ( $2.5^{\prime}$ and $4.3^{\prime}$, respectively), in the sense of a steepening with increasing radius as expected from two-body relaxation. It would be useful to determine the mass function, in a similar way, at an even greater distance from the cluster center in order to determine whether this steepening continues into the outer halo. Given the sharp drop of stellar surface density in the halo, this may require larger area, ultradeep, ground-based imaging to obtain sufficient statistics.

Radial velocities alone do not strongly constrain the anisotropy profile. Both radially biased and tangentially biased orbit distributions are capable of producing a halo flattening of the projected velocity dispersion profile. In contrast, the anisotropy profile can be directly determined from proper motions, either alone (e.g., Cudworth 1979 for M3) or in conjunction with radial velocities (e.g., Lupton et al. 1987 for M13). In the case of M92, Cudworth (1979) finds little evidence for anisotropy, although it appears that this data set would only be capable of detecting a strong signal.

Testa et al. (2000) have used the Digitized Palomar Observatory Sky Survey to examine the spatial structure of M92 near and beyond the $\sim 15^{\prime}$ tidal radius. They found clear evidence for a flattening of the profile beyond their best-fit tidal radius of $r_{t}=$ $12.3^{\prime}$, which they interpret as a extratidal halo that extends to approximately $30^{\prime}$. The structure of this halo is essentially circular, showing at best weak evidence for the sort of bipolar elongation ("tails") seen in some other clusters (e.g., Palomar 5; Odenkirchen et al. 2001). Testa et al. (2000) suggest that this may indicate that the extratidal halo of M92 consists of stars evaporating from the cluster that have not yet formed an escaping tidal stream. This would tend to argue that two-body relaxation and possibly tidal shocking dominate over tidal stresses in determining the halo structure and mass-loss rate in M92.

Improved dynamical cluster models, which take advantage of the growing data sets provided by high-resolution imaging and spectroscopy, should provide a more sensitive measure of the role of tidal influences on cluster halos. This will lead to improved estimates of cluster mass-loss rates and dissolution timescales.

We would like to thank Doug Geisler for his advice on using the Washington system and Heather Morrison for making her data available to us. G. A. D. was supported in part by NASA LTSA grant NAG 5-6404 and, during the completion of this study, by grants for the following two HST projects: Support for programs GO-9839 and AR-10292 was provided by NASA through a grant from the Space Telescope Science Institute, which is operated by the Association of Universities for Research in Astronomy, Inc., under NASA contract NAS5-26555. The GRAPE simulations were supported by NASA ATP grant NAG 5-2781 to Indiana University and also received generous support from Indiana University Information Technology Services. S. S. acknowledges the support of the School of Engineering, Mathematics and Science at Purdue University Calumet for publication costs.

\section{REFERENCES}

Aarseth, S. J. 1999, PASP, 111, 1333

Andreuzzi, G., Buonanno, R., Fusi Pecci, F., Iannicola, G., \& Marconi, G. 2000, A\&A, 353, 944

Barnard, E. E. 1931, Publ. Yerkes Obs., 6

Beers, T. C., Preston, G. W., Schectman, S. A., \& Kage, J. A. 1990, AJ, 100, 849

Binney, J., \& Tremaine, S. 1987, Galactic Dynamics (Princeton: Princeton Univ. Press)

Buonanno, R., Buscema, G., Corse, C. E., Iannicola, G., Smriglio, F., \& Fusi Pecci, F. 1983, A\&AS, 53, 1

Canterna, R. 1976, AJ, 81, 228

Chaboyer, B., Demarque, P., \& Sarajedini, A. 1996, ApJ, 459, 558

Combes, F., Leon, S., \& Meylan, G. 1999, A\&A, 352, 149

Cudworth, K. M. 1976, AJ, 81, 975 1979, AJ, 84, 1312

Dehnen, W., Odenkirchen, M., Grebel, E. K., \& Rix, H. 2004, AJ, 127, 2753 Drukier, G. A., Bailyn, C. D., van Altena, W. F., \& Girard, T. M. 2003, AJ, 125, 2559 Drukier, G. A., Cohn, H. N., Lugger, P. M., \& Yong, H. 1999, ApJ, 518, 233

Drukier, G. A., Slavin, S. D., Cohn, H. N., Lugger, P. M., Berrington, R. C., Murphy, B. W., \& Seitzer, P. O. 1998, AJ, 115, 708 (Paper I)

Gebhardt, K., Pryor, C., O’Connell, R. D., Williams, T. B., \& Hesser, J. E. 2000, AJ, 119, 1268

Gebhardt, K., Pryor, C., Williams, T. B., \& Hesser, J. E. 1994, AJ, 107, 2067

Geisler, D. 1984, PASP, 96, 723 1996, AJ, 111, 480

Geisler, D., Clariá, J. J., \& Minniti, D. 1991, AJ, 102, 1836

Gerssen, J., van der Marel, R. P., Gebhardt, K., Guhathakurta, P., Peterson, R. C., \& Pryor, C. 2002, AJ, 124, 3270

Gnedin, O. Y., Lee, H. M., \& Ostriker, J. P. 1999, ApJ, 522, 935

Good, I. J. 1950, Probability and the Weighing of Evidence (New York: Hafner Publ. Co.)

Gunn, J. E., \& Griffin, R. F. 1979, AJ, 84, 752

Harris, H. C., \& Canterna, R. 1979, AJ, 84, 1750

Harris, W. E. 1996, AJ, 112, 1487

Jaynes, E. T. 2003, Probability Theory: The Logic of Science (New York: Cambridge Univ. Press)
Johnston, K. V., Sigurdsson, S., \& Hernquist, L. 1999, MNRAS, 302, 771

King, I. B. 1966, AJ, 71, 64

Lasker, B. M., Sturch, C. R., McLean, B. J., Russell, J. L., Jenkner, H., \& Shara, M. M. 1990, AJ, 99, 2019

Lupton, R., Gunn, J. E., \& Griffin, R. F. 1985, in IAU Symp. 113, Dynamics of Star Clusters, ed. J. Goodman \& P. Hut (Dordrecht: Reidel), 19 . 1987, AJ, 93, 1114

MacKay, D. J. C. 2003, Information Theory, Inference, and Learning Algorithms (New York: Cambridge Univ. Press)

Makino, J., Taiji, M., Ebisuzaki, T., \& Sugimoto, D. 1997, ApJ, 480, 432

Nassau, J. J. 1938, ApJ, 87, 361

Norris, J., \& Zinn, R. 1977, ApJ, 215, 74

Odenkirchen, M., et al. 2001, ApJ, 548, L165

Pryor, C., Latham, D., \& Hazen, M. 1988, AJ, 96, 123

Ratnatunga, K. U., \& Freeman, K. C. 1989, ApJ, 339, 126

Rees, R. F., Jr. 1992, AJ, 103, 1573

Sandage, A., \& Walker, M. F. 1966, ApJ, 143, 313

Sawyer Hogg, H. 1973, Publ. David Dunlap Obs., 3, 6

Scarpa, R., Marconi, G., \& Gilmozzi, R. 2003, A\&A, 405, L15

. 2006, in AIP Conf. Proc. 822, First Crisis in Cosmology Conference, ed. E. J. Lerner \& J. B. Almeida (New York: AIP), 102

Sivia, D. S. 1996, Data Analysis: A Bayesian Tutorial (Oxford: Oxford Univ. Press)

Soderberg, A. M., Pilachowski, C. A., Barden, S. C., Willmarth, D., \& Sneden, C. 1999, PASP, 111, 1233

Sosin, C., \& King, I. R. 1997, AJ, 113, 1328

Stetson, P. B. 1987, PASP, 99, 191

Stetson, P. B., \& Harris, W. E. 1988, AJ, 96, 909

Takahashi, K., \& Portegies Zwart, S. F. 2000, ApJ, 535, 759

Testa, V., Zaggia, S. R., Andreon, S., Longo, G., Scaramella, R., Djorgovski, S. G., \& de Carvalho, R. 2000, A\&A, 356, 127

Tonry, J., \& Davis, M. 1979, AJ, 84, 1511

Trager, S. C., King, I. R., \& Djorgovski, S. 1995, AJ, 109, 218

Tucholke, H.-J., Scholz, R.-D., \& Brosche, P. 1996, A\&A, 312, 74

Zinn, R. J., Newell, E. B., \& Gibson, J. B. 1972, A\&A, 18, 390 